This item was submitted to Loughborough's Institutional Repository (https://dspace.lboro.ac.uk/) by the author and is made available under the following Creative Commons Licence conditions.

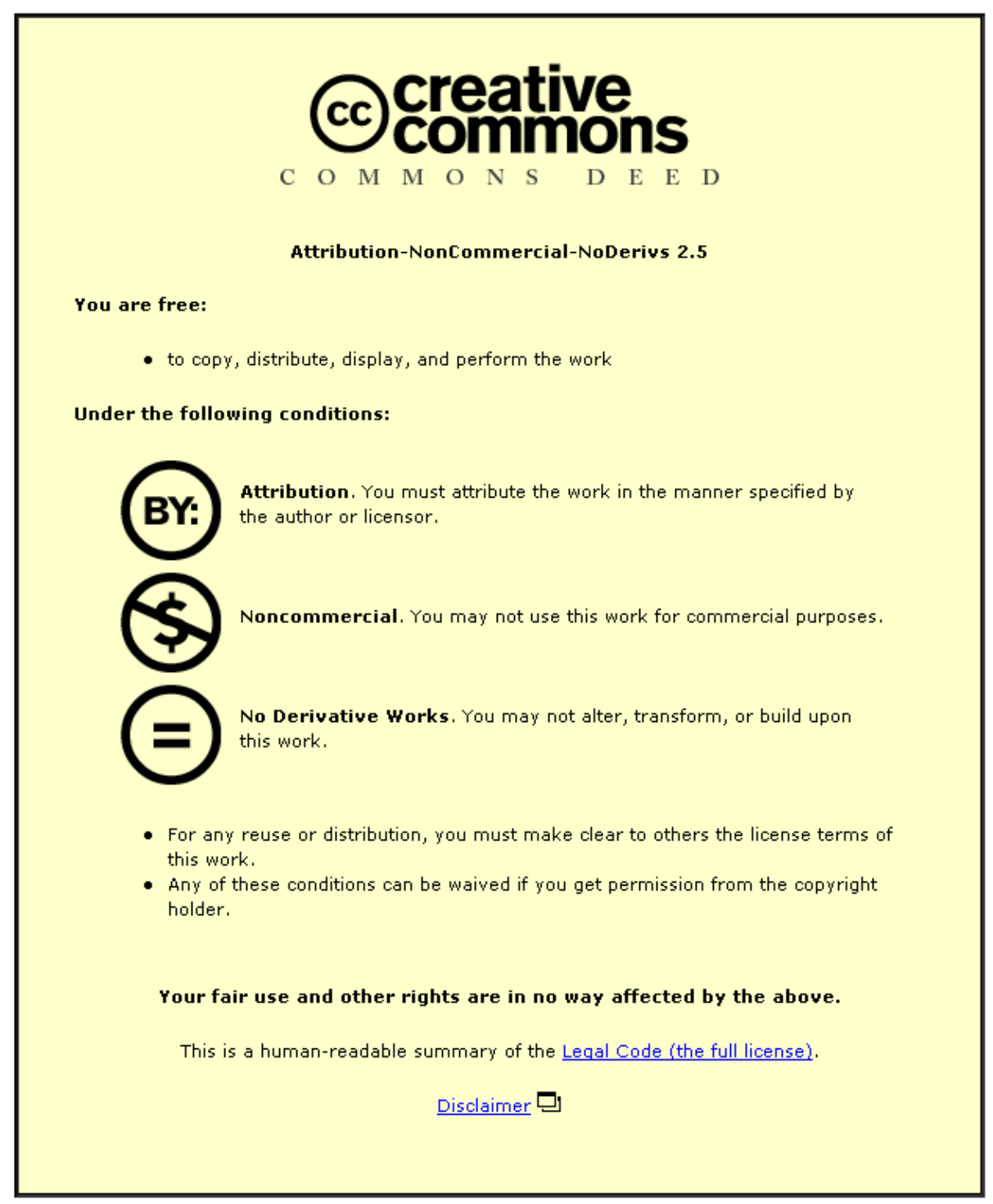

For the full text of this licence, please go to: http://creativecommons.org/licenses/by-nc-nd/2.5/ 


\title{
Aeronautical Charging Policy Incentive Schemes for Airlines at European Airports
}

\author{
Owain Cai Jones, Lucy C.S. Budd and D. E. Pitfield*, \\ Transport Studies Group, \\ School of Civil \& Building Engineering, \\ Loughborough University, \\ Loughborough, LE11 3TU \\ U.K. \\ *Corresponding author D.E.Pitfield@lboro.ac.uk
}

\begin{abstract}
This paper introduces the concept of incentive schemes that may accompany airports' aeronautical charging policies and develops a taxonomy of such schemes based on an analysis of data for 46 European Airports held in the RDC Aviation database. ${ }^{1}$ This taxonomy details the different types of incentive schemes that in are operation. It is clear their use is widespread and that the magnitude of the incentive is often significant. A financial benchmarking analysis is undertaken for four selected airports to illustrate the extent of the variations between airports both in terms of the basic characteristics of the incentive schemes and in the periods over which the discounts are available.
\end{abstract}

\section{Introduction}

The deregulation and liberalisation of the European aviation industry during the 1990's (Barrett, 2000) has caused European airports to evolve from being merely infrastructure providers into fully fledged businesses in their own right (International Air Transport Association, hereafter IATA, 2012). In 2009, 80\% of European airports operated as commercial entities, with nearly half of European air passengers travelling through fully or partially privatised airports (IATA, 2012). The shift towards whole or partial privatisation and commercialisation has resulted in airports using revenues to cover costs and, ideally, making a return on their investments. The revenue generated within a privatised airport is typically derived from two distinct revenue streams: aeronautical and non-aeronautical charges.

Charges that are levied on aircraft operators for the use of airport facilities and services are known as "aeronautical charges". The revenue generated through aeronautical charges belongs to the aeronautical revenue stream. Landing charges (usually based on aircraft weight), passenger (PAX) charges and aircraft parking charges are the usual aeronautical charges charged by airports. This is in contrast to non-aeronautical charges that are derived from commercial activities conducted within the airport grounds (e.g. car parking fees and rents from retail concessions) (Fichert \& Klophaus, 2011). According to IATA, 47\% of European airport revenues during 2009 were derived from nonaeronautical sources and 53\% from aeronautical sources (IATA, 2012).

Due to the increasingly competitive nature of the airline and airport industry, a number of airports offer discounts and / or financial or other incentives (known as "Incentive Schemes") to entice airlines into offering new or expanding existing services. The targets of these schemes are airlines seeking to achieve cost reductions (Barrett, 2000). This paper aims to discover how widespread the use of incentive schemes is within a sample of European airports and the types of incentives that are offered. A taxonomy of incentive schemes used by 46 European airports is developed and a benchmarking exercise, to determine the financial implications of a select number of incentive schemes, is conducted.

\footnotetext{
${ }^{1}$ www.rdcaviation.com
} 


\section{Aeronautical Charges and Incentive Schemes}

Fichert andKlophaus (2011) provide a usual overview of the main types of incentive scheme that an airport may offer (see Figure 1).

\section{Figure 1 near here}

They highlight an important difference between an incentive that sits within an established system and a policy that offers separate incentives.

The incentives listed on the right hand side of Figure 1 are known within the industry as being separate incentive schemes for which a clearly defined target / objective must be met for the reward to be delivered. The objectives of extant incentive schemes can vary considerably, from general criteria such as increasing annual Maximum Take-Off Mass $^{2}$ (MTOM) to specific growth criteria for a particular route. Due to the wide scope and combination possibilities of incentive schemes, it is up to the management teams within individual airports (or groups of airports) to devise appropriate incentive schemes that encourage airline operators to deliver the objectives that the airport desires. This creative freedom is responsible for producing the vast array of incentive schemes that are in use today. For example, Malta International Airport has chosen to waive all landing fees for the 2011/2 winter period (Times of Malta, 2011) while Avinor AS-operated airports offer weekly discount cards to certain types of operation.

In an area of depressed margins and intense competition, the availability of these incentive schemes is becoming increasingly important for airlines. Approximately $3.5-5 \%$ of an airlines' total cost is directly related to airport charges (Barrett, 2000; Airports Council International, 2007; Airports Council International-E(a), 2010) and so any way of reducing this cost burden is often enthusiastically embraced. However, no research has examined the content of these incentive schemes or provided a taxonomy of their defining features. This paper addresses this gap and analyses the possible financial implications of selected incentive schemes to airlines.

\section{A Taxonomy of European Airports}

All commercial airports within Europe are classified by Airports Council International (ACI) into four groups according to how many passengers they handle a year. The four groups are:

- $\quad$ Group 1 (I): Airports with more than 25 million PAX per annum. Within this group one can typically find such airports as London Heathrow (LHR) and Frankfurt (FRA),

- $\quad$ Group 2 (II): Airports with between 10 and 25 million PAX per annum, including airports such as London Stansted (STN) and Zürich (ZRH),

- $\quad$ Group 3 (III): Airports with between 5 and 10 million PAX per annum, with airports such as Birmingham (BHX) and Lyon (LYS) included within this group, and

- $\quad$ Group 4 (IV): Airports with less than 5 million PAX per annum, including airports such as Bournemouth International (BOH) and Frankfurt-Hahn (HHN). (ACI-E, 2011).

These groups are dynamic, and airports can move between groups as passenger traffic grows or declines. The groups are published ${ }^{3}$ on a monthly basis, with a typical "lag time" of up to one month. At the beginning of each new calendar year an annual summary statement of the previous calendar year is published, along with the data for the calendar month of December. Data for the full calendar year of 2010 (the most recent available) was used for this research.

\footnotetext{
${ }^{2}$ Maximum Take-Off Weight (MTOW) is more commonly used than MTOM within the industry. It is up to the airport to decide whether to use MTOW or MTOM.

${ }^{3}$ ACI-E’s monthly “Airport Traffic Research” which is published online for member airports.
} 
Rather than focus on the charging schemes that are offered by the busiest passenger airports in Europe (which may not need to offer such extensive incentive schemes because of their historical and contemporary importance as major international hubs), this research will focus on the incentive schemes that are offered by the smallest airports in Group IV that are very keen to develop the network of routes served from their facility and increase passenger throughput.

The 27 airports that are within ACI-E’s group IV are:

\section{Table 1 near here}

The annual PAX figures for this group range from 5,026,976 per annum (IBZ) to 296,726 (LGG) and are located within a range of different European countries. However, while the group IV is useful for identifying airports handling similar passenger numbers, the ACI-E classification does not take into account the traffic mix and relative status (i.e. major hub, primary/secondary gateway) of the airports concerned. This is a potentially serious omission and consequently, it was necessary to add other comparable airports to the original list. The additions were informed by the findings of internal benchmarking exercises that were performed by one of the airports in Group 4 to identify not only their major competitors but also the airports to which they aspired ${ }^{4}$. The full list of 46 airports whose incentive schemes were examined for this research is provided in Table 2.

\section{Table 2 near here}

The PAX traffic throughput noted within these airports now range from 53,009,221 (FRA) to 296,726 (LGG).

Data on the existence and content of the aeronautical charging and separate incentive schemes for the 46 airports was derived from RDC Aviation's Airport Charges database. RDC Aviation is a commercial Nottingham (U.K.) based company who specialise in aviation consultancy, data gathering and aviation software systems. Their Airport Charges database contains over 2,000 documents relating to aeronautical charging policies of airports worldwide (RDC Aviation, 2012). These documents contain data on all airport aeronautical policies including (but not limited to), landing fees, noise and emissions charges, infrastructure levies, charges for aircraft parking and the use of airbridges, terminal and en route navigation charges, passenger and PRM (persons with restricted mobility) charges, security charges, government and other state taxes, and fuel prices (RDC Aviation, 2012).

All 46 airports in our sample published an aeronautical charging policy. For each airport, the aeronautical charging policy was accessed and saved. An analysis of the content/focus of each scheme was then performed to enable both generic and specific information about each one to be captured. A minimum of nine pieces of information about each scheme was recorded, the details of which appear in Table 3. Of the 46 airports in the sample, 40 operated a separate incentive scheme and 33 of these schemes were unique.

\footnotetext{
${ }^{4}$ For reasons of commercial confidentiality, we have not identified the airport.
} 


\section{Table 3 near here}

\section{Key findings}

Interestingly, while numerous different schemes are used by the 46 airports, the following airports operated exactly the same incentives:

•BRI, BDS (“Aeroporti Di Puglia” controlled airports), NAP

- HAU, BGO, AES, BOO, TRD, TOS, SVG, KRS (“Avinor AS” controlled airports)

• IBZ, SVQ, VLC, AGP, LPA, ALC, PMI, FUE, BIO, TFN, GRO (“Aena” controlled airports)

The analysis showed that incentive schemes primarily vary by the duration of their operation (Table 4) and by their primary focus (Table 5).

\section{Table 4 and 5 near here}

Noteworthy incentive schemes include

- AMS, whose scheme stated it offers a "reward" if criteria are met, but the nature of the "reward" was not specified.

- HAJ allows individual airline operators to decide which charge they would like to be discounted.

- $\quad$ PRG offers free use of the airports airbridges if certain growth criteria are met.

- TRN charges different levies depending if the destination and whether the flight serves an "EU" or “extra EU” airport.

- MMX and BMA discount certain fees, but do not give details as to which ones.

- MUC imposes a surcharge for polluting aircraft, using the ERLIG formula ${ }^{5}$ :

Other special conditions included exemptions for:

- Operations exclusively made for the transportation of state or government heads and ministers, and reciprocity agreements, confirmed by the national Foreign Office (or equivalent).

- $\quad$ Operations by military aircraft or others, performing official military missions.

- $\quad$ Operations for search and rescue, medical emergencies, internal security, civil protection and humanitarian missions.

- $\quad$ Aircraft returning back to the airport due to a technical breakdown, weather conditions or other cases of force majeure.

Clearly, the use of incentive schemes is extensive, and their scope wide ranging. In order to determine the possible financial implications for airlines of these different schemes, a financial benchmarking exercise of four carefully selected incentive schemes, Malta (MLA), Birmingham UK (BHX), Brussels (BRU), and Nice (NCE), was conducted.

\section{Financial Benchmarking}

All four airports operate an Incentive Scheme which is not built into the standard aeronautical charging policy. To ensure comparability, the aircraft type, operating period (summer / winter), flight type

\footnotetext{
${ }^{5}$ ERLIG stands for "Emissions Related Landing charges Investigation Group". A group founded by the 2001 European Civil Aviation Conference (ECAC) to develop a charging model (Federal Office of Civil Aviation, 2001
} 
(domestic / intra-Europe / International), turn-around time and extras (such as airbridges) will be constant and consistently applied to all four schemes.

The total aeronautical costs involved with operating a particular aircraft type into each airport is calculated and then reduced in line with each airport's incentive scheme.

The RDC Aviation Hub web-page features an inbuilt tool that can be used for benchmarking purposes. This tool is effectively a calculator that enables the computing of written aeronautical charging polices into numerical data. However, incentive scheme data is not computed automatically. To operate the tool, the user must first select the airport of interest, and then state the characteristics of the flight that will use the airport. If the use of an Incentive Scheme is also to be included then the user must manually insert this data into the tool by selecting which item is to be discounted and by how much (in percent). Once the input of this data is complete a page detailing the costs likely to be incurred by the airline are shown.

The analysis of the incentive schemes operated by the four airports posed various intriguing lines of enquiry relating to the benefits that could be accrued over time and whether the schemes favoured particular aircraft types (it could be hypothesised, for example, that airports targeting B737NG operators, for example, were hoping to attract a particular major low cost airline). Three different aircraft types were selected, the Boeing 737-800 (B738) with a theoretical maximum seating capacity of 189 passengers, the Airbus A330-300 (A333) with 221 seats and the Airbus A340-300 (A343) with 247 seats. The seating capacities were based on the configurations flown by Ryanair (for the B738) and Lufthansa (for the A333 and A343), currently two of the biggest scheduled airlines in Europe (Lufthansa, 2012, Ryanair, 2012,).

As the Incentive Schemes are influenced by PAX Seat Load Factor (SLF), an industry standard ${ }^{6}$ SLF of $70 \%$ was selected. This had the result that, when applied to the three aircraft types:

- $\quad$ Ryanair’s B738 with a MTOW of 66.99 tonnes now carries 132 PAX instead of 189,

- $\quad$ Lufthansa's A333 with a MTOW of 233 tonnes, carries 155 PAX instead of 221, and

- $\quad$ Lufthansa’s A343 with a MTOW of 271 tonnes, carries 173 PAX instead of 247.

With the airframe and the aircraft configurations chosen, the flight characteristics were selected. The B738 is predominantly used for intra-EU flights by Ryanair so the B738 flights in this exercise will all be assumed to operate EU services. As some airports operate a different incentive policy for the winter and summer periods (such as MLA), both seasons will be assessed. The summer period will be described as the "generic" incentive package and the winter period the "strategic" scheme. This is due to the strategic importance of smoothing the "seasonality effect" within airport PAX figures. Additional extras, such as airbridges will be added to Lufthansa's (A333 and A343) aircraft operations but not to Ryanair's B738 ones, as per normal operating procedures for the airlines. Government taxation will not be included in the analysis as this does not go to the airport.

The raw computations for the benchmarking exercise are provided in Tables 6-11 in the sequence of summer and winter operations for the A343, A333, and B738. Figures 2 to 7 display this raw information in a graphical format. The bar charts are colour coded to represent each individual airport (blue represents MLA, red represents NCE, et cetera), the first bar for each airport represents a "zero year". This is included so a comparison can be made between the normal full aeronautical charge (i.e. without any discount applied), and the cost when the incentive scheme is applied. The subsequent bars represent each subsequent year, so the second bar represents the first year of operation under the

\footnotetext{
${ }^{6}$ Known through work performed whilst at Malta International Airport plc
} 
incentive scheme. When the bar returns back to the same level noticed in the "zero year" it indicates that the operational timeframe of the incentive scheme has finished. If the bar does not return to the "zero year", as is with the case of BHX, the incentive scheme is in place for a period of four or more years.

\section{Tables 6-11 near here Figures 2-7 near here}

As one would expect, the larger the airframe, the higher the aeronautical charge that is levied by all the airports. The airport that charged the highest "year zero" rate for both the Airbus airframes was BHX, charging €6,389.00 (an average of €36.93 per departing PAX) for the LH A340-300 and €5,579.00 (€35.99 average per PAX) for the LH A330-300. BRU charged the highest "year zero" rate for the FR B737-800, charging €3,995.00 (an average of €30.27 per PAX). The airport that charged the lowest "year zero" aeronautical charge was MLA, charging an average of €3,632.00 or €23.50 per PAX.

The highest discount (in monetary terms) was given by BHX, who offered a discount of $€ 4,359.00$ (68.23\%) on the first year of the strategic incentive when operating a LH A340-300. When the four years of operating a single flight within the BHX strategic incentive programme is compared with the "zero year" it is evident that significant cost reductions are possible. If an airline operated a Lufthansa-configured A340-300 once a year from BHX under the normal aeronautical charging policy, the total charge would be $€ 25,556.00$. However, if the same operation was charged under the strategic incentive policy, then the total charge would be reduced by $€ 11,070.00$ (total cost $€ 14,486.00)$.

The lowest discount in terms of total amount saved is offered by NCE under the generic incentive scheme. This scheme lasts for only two years and would reduce aeronautical charges for a FR B737800 operation by only $€ 1,101.00$ (from $€ 5,794.00$ to $€ 4,693.00$ ).

The Incentive Schemes from MLA, NCE and BHX display a clear pattern of annual reductions in the incentive discount that is offered. The graphs show that in subsequent years the amount charged by the airport to the airline gradually increases until the standard charge is realised, normally after three or four years. The Incentive Scheme at BRU differs from this pattern. If an airline were to operate under the strategic Incentive Scheme at BRU, it can expect to be charged an average discount of approximately $31 \%$ for a three year period.

All of the Incentive Schemes included within the benchmark reduced their standard passenger charge. The discount varied depending if the strategic or generic scheme was in use by the airline. The discount offered also varied depending on the structure of the standard aeronautical charging policy. The airports with a higher emphasis on charging for aircraft related levies ${ }^{7}$ (NCE and BHX) offered a lower discount on $\mathrm{PAX}^{8}$ related levies. However, these airports also offered discounts on aircraft related levies, whereas the airports with the main emphasis on charging for PAX related services (MLA and BRU) did not.

\footnotetext{
${ }^{7}$ Includes levies for: Landing, Environment, Terminal Navigation, Infrastructure, Airbridge and Aircraft Parking charges.

${ }^{8}$ Includes levies for: PAX, Security and Infrastructure charges.
} 
Birmingham International Airport's strategic Incentive Scheme policy seems to encourage the operation of an airframe in the FR B737-800 configuration. Although this was not the highest incentive given by BHX the incentive is very noticeable when compared with the other airports included within the benchmarking exercise. This is especially evident when reviewing the strategic incentive policies. The percentage discount offered during the first year of operation amounts to $79.1 \%$, saving the aircraft operator $€ 2,016.00$ per aircraft movement. This reduces the "per PAX" amount from $€ 21.24$ (the median average for this "year zero" benchmark being €21.60), to an outstandingly low amount of $€ 5.97$. The generic Incentive Scheme also seems to favour this type of aircraft operation which is achieved by offering very attractive discounts on levies, and implementing a non-standard pattern of annual reductions. This setup, in essence, aims to encourage the aircraft operator to continue services for at least three years.

\section{Conclusion}

The aim of this paper was to identify the different types of incentive scheme that are used by a sample of European airports and then, with the aid of a benchmarking exercise, analyse the financial implications of a select number of these schemes.

While the majority of airports surveyed offered a discount on their standard aeronautical charging policy, the nature of the discounts varied. Once the differences for domestic and international flights were excluded, reductions in passenger and runway/landing fees were the most popular items to discount. However, only 33 schemes were unique, with two airport authorities "Avinor AS" and "Aena”) applying the same aeronautical charging polices at a number of their airports. A number of interesting incentive schemes were identified, including the "select your own discount" offered by Hanover. As well as recognising some of the commonalities in incentive schemes that were identified by Graham (2008) and Fichert \& Klophaus (2011) a number of previously unknown traits were also discovered. This particularly related to aircraft operations that are exempt from the aeronautical charging policy, for example at “Aena” and "Avinor AS” controlled airports.

Regarding the financial benchmarking exercise, the precise nature of the financial discounts on offer to selected operators of particular aircraft types were computed. This is the first known research into the financial implications of these incentive schemes. It was discovered that the financial rates offered by incentives varied between the four airports (MLA, BRU, NCE \& BHX). The variations were found to differ between strategic and generic aircraft operations, with each airport offering an increased incentive to perform flights to specific locations and / or during the winter period. Each airport also gave a discount for all new aircraft operations (generic incentive), which was found to be lower than that of the strategic incentive scheme. Similarities were also found to exist within the time frame and discounts offered by the incentive schemes, with the percentage discount on offer reducing annually.

The incentive scheme with the longest operational lifetime was Birmingham International Airport's, which lasted four years. This airport also offered the highest recorded financial incentive at $€ 4,359.00$ (for LH A340-300 operations), and offered a 79.1\% discount on aeronautical fees for FR B737-800 operations within the first year of the strategic incentive scheme. The lowest discount in terms of total amount saved is offered by NCE under the generic incentive scheme. This scheme last for only two years and would reduce aeronautical charges for a single annual FR B737- 800 operation by only $€ 1,101.00$, from $€ 5,794.00$ to $€ 4,693.00$. The importance of such schemes to airlines is likely to grow and, as increasing numbers of airports compete for airline custom, it is not unreasonable to suggest that they might become ever-more important marketing devices for airports and bargaining tools for airlines who are seeking to reduce their operating costs to remain competitive. 


\section{References}

Airports Council International, 2007, “The Airport Business”, Position Brief. Airports Council International, Montréal.

Airports Council International-E, 2010(a), “Economics Report 2010”, Airports Council International - Europe, Brussels.

Airports Council International -E, 2011, Airport Traffic Report - December 2010 \& Full Year, Airports Council International - Europe, Brussels.

Barrett, S. 2000, Airport competition in the deregulated European aviation market, Journal of Air Transport Management, 6, 13-27.

Fichert, F. and Klophaus, R. 2011, Incentive schemes on airport charges - Theoretical analysis and empirical evidence from German airports, Research in Transport \& Management, 1, 71-79.

Graham, A. 2008, “Managing airports. An international perspective” ( $3^{\text {rd }}$ ed). Oxford: Butterworth Heinemann

International Air Transport Association, 2012. Fast facts. [online] Available at: < http://www.acieurope.org/policy/fast-facts.html> [Accessed 19 March 12].

Lufthansa,2012. "Lufthansa -Traffic Figures". [online] Available at

$<$ http://investor-relations.lufthansa.com/en/finanzberichte/traffic-figures.html> [Last Accessed: 5 April 2012]

RDC Aviation. 2012, Airport Charges Data from airportcharges.com, RDC Aviation limited. [online]. Available at: <www.rdcaviation.com/software/airportcharges> [Last accessed: 1 April 2012] 
Table 1: Airports included within ACI-E 2010 group IV.

\begin{tabular}{lllll}
\hline Airport & IATA Airport & IATA & Airport & IATA \\
Aalesund & AES Girona & GRO & Porto & OPO \\
Aberdeen & ABZ Gothenburg & GOT & Riga & RIX \\
Bergen & BGO Hahn & HHN & Sevilla & SVQ \\
Bilbao & BIO Haugesund & HAU & Stavanger & SVG \\
Bodo & BOO Ibiza & IBZ & Stockholm & BMA \\
Bordeaux & BOD Kristiansand & KRS & Tenerife & TFN \\
Brindisi & BDS Liege & LGG & Tromsoe & TOS \\
Dubrovnik & DBV Malmo & MMX & Trondheim & TRD \\
Fuerteventura & FUE Malta & MLA & Turin & TRN \\
\hline
\end{tabular}

Source: ACI-E, 2011

Table 2: Airports included within ACI-E group IV with the additional benchmarked airports.

\begin{tabular}{llll}
\hline Airport & IAT Airport & IAT Airport & IAT Airport IATA \\
Aalesund & AES Frankfurt & FRA Malmo & MM Tromso TOS \\
Aberdeen & ABZ Fuerteventura & FUE Malta & MLA Trondhei TRD \\
Alicante & ALC Girona & GRO Manchester & MANTurin TRN \\
Amsterdam & AMS Gothenburg & GOT Munich & MUC Valencia VLC \\
Athens & ATH Gran Canaria & LPA Naples & NAP \\
Bari & BRI Hahn & HHN Nice & NCE \\
Bergen & BGO Hanover & HAJ Palma, Mallorca & PMI \\
Bilbao & BIO Haugesund & HAU Porto & OPO \\
Birmingham & BHX Ibiza & IBZ Prague & PRG \\
Bodo & BOO Kristiansand & KRS Riga & RIX \\
Bordeaux & BOD Liege & LGG Sevilla & SVQ \\
Brindisi & BDS Lisbon & LIS Stavanger & SVG \\
Brussels & BRU London & LGW Stockholm & BMA \\
Dubrovnik & DBV Malaga & AGP Tenerife & TFN \\
\hline
\end{tabular}

Source of airport data: ACI-E (2011). 
Table 3: Details of the 46 airport incentive schemes

\begin{tabular}{|c|c|c|c|c|c|c|c|c|c|}
\hline & New Destinations & Specific Target & Increased Feq. & Off Peak & On Peak & Item Discounted & Duration & Min. Requirements & Special Offer \\
\hline $\begin{array}{l}\text { GRO } \\
\text { Summer } \\
\text { Winter } \\
\text { Off Peak } \\
\end{array}$ & $\begin{array}{l}\mathrm{N} \\
\mathrm{N} \\
\mathrm{Y} \\
\end{array}$ & $\begin{array}{l}\mathrm{Y} \\
\mathrm{Y} \\
\mathrm{Y} \\
\end{array}$ & $\begin{array}{l}\mathrm{N} \\
\mathrm{N} \\
\mathrm{N} \\
\end{array}$ & $\begin{array}{l}\mathrm{Y} \\
\mathrm{Y} \\
\mathrm{Y} \\
\end{array}$ & $\begin{array}{l}\mathrm{Y} \\
\mathrm{Y} \\
\mathrm{Y} \\
\end{array}$ & Different fees if destination is Intemational or Domestic & Constant & Depending if international or domestic & (2) \\
\hline $\begin{array}{l}\text { GOT } \\
\text { Summer } \\
\text { Winter } \\
\text { Off Peak }\end{array}$ & $?$ & $?$ & $?$ & $?$ & $?$ & $?$ & $?$ & new routes to destinations that have not been served during the last 12 months & $\mathrm{NA}$ \\
\hline $\begin{array}{l}\text { BDS } \\
\text { Summer } \\
\text { Winter } \\
\text { Off Peak }\end{array}$ & $\begin{array}{l}\mathrm{N} \\
\mathrm{N} \\
\mathrm{Y}\end{array}$ & $\begin{array}{l}\mathrm{Y} \\
\mathrm{Y} \\
\mathrm{Y}\end{array}$ & $\begin{array}{l}\mathrm{N} \\
\mathrm{N} \\
\mathrm{N} \\
\mathrm{N}\end{array}$ & $\begin{array}{l}\mathrm{Y} \\
\mathrm{Y} \\
\mathrm{Y}\end{array}$ & $\begin{array}{l}\mathrm{Y} \\
\mathrm{Y} \\
\mathrm{Y} \\
\end{array}$ & Different fees if destination is Intemational or Domestic & Constant & Depending if intermational or domestic & N/A \\
\hline $\begin{array}{l}\text { OPO } \\
\text { Summer } \\
\text { Winter } \\
\text { Off Peak }\end{array}$ & $\begin{array}{l}\mathrm{N} \\
\mathrm{N} \\
\mathrm{Y}\end{array}$ & $\begin{array}{l}\mathrm{Y} \\
\mathrm{Y} \\
\mathrm{Y}\end{array}$ & $\begin{array}{l}\mathrm{N} \\
\mathrm{N} \\
\mathrm{N}\end{array}$ & $\begin{array}{l}\mathrm{Y} \\
\mathrm{Y} \\
\mathrm{Y}\end{array}$ & $\begin{array}{l}\mathrm{Y} \\
\mathrm{Y} \\
\mathrm{Y}\end{array}$ & $\begin{array}{l}\text { Different fees if destination is Schengen or EU non- } \\
\text { Schengen or International }\end{array}$ & Constant & Rate is discounted depending on destination "band" & Reduction for test and training flights of $50 \%$ \\
\hline $\begin{array}{l}\text { IBZ } \\
\text { Summer } \\
\text { Winter } \\
\text { Off Peak }\end{array}$ & $\begin{array}{l}\mathrm{N} \\
\mathrm{N} \\
\mathrm{Y}\end{array}$ & $\begin{array}{l}\mathrm{Y} \\
\mathrm{Y} \\
\mathrm{Y}\end{array}$ & $\begin{array}{l}\mathrm{N} \\
\mathrm{N} \\
\mathrm{N}\end{array}$ & $\begin{array}{l}\mathrm{Y} \\
\mathrm{Y} \\
\mathrm{Y}\end{array}$ & $\begin{array}{l}\mathrm{Y} \\
\mathrm{Y} \\
\mathrm{Y}\end{array}$ & $\begin{array}{l}\text { Different fees if destination is within EU, Domestic or } \\
\text { Inter-Islands }\end{array}$ & Constant & Rate is discounted depending on destination "band" & $\mathrm{N} / \mathrm{A}$ \\
\hline $\begin{array}{l}\text { RIX } \\
\text { Summer } \\
\text { Winter } \\
\text { Off Peak }\end{array}$ & $\begin{array}{l}\mathrm{N} \\
\mathrm{N} \\
\mathrm{N}\end{array}$ & $\begin{array}{l}\mathrm{N} \\
\mathrm{N} \\
\mathrm{N}\end{array}$ & $\begin{array}{l}\mathrm{N} \\
\mathrm{N} \\
\mathrm{N}\end{array}$ & $\begin{array}{l}\mathrm{N} \\
\mathrm{N} \\
\mathrm{N}\end{array}$ & $\begin{array}{l}\mathrm{N} \\
\mathrm{N} \\
\mathrm{N}\end{array}$ & N/A & N/A & N/A & Training flights use coefficient of 0.75 \\
\hline $\begin{array}{l}\text { SVQ } \\
\text { Summer } \\
\text { Winter } \\
\text { Off Peak }\end{array}$ & $\begin{array}{l}\mathrm{N} \\
\mathrm{N} \\
\mathrm{Y}\end{array}$ & $\begin{array}{l}\mathrm{Y} \\
\mathrm{Y} \\
\mathrm{Y}\end{array}$ & $\begin{array}{l}\mathrm{N} \\
\mathrm{N} \\
\mathrm{N}\end{array}$ & $\begin{array}{l}\mathrm{Y} \\
\mathrm{Y} \\
\mathrm{Y}\end{array}$ & $\begin{array}{l}\mathrm{Y} \\
\mathrm{Y} \\
\mathrm{Y}\end{array}$ & $\begin{array}{l}\text { Different fees if destination is within EU, Domestic or } \\
\text { Inter--slands }\end{array}$ & Constant & Rate is discounted depending on destination "band" & N/A \\
\hline $\begin{array}{l}\text { DBV } \\
\text { Summer } \\
\text { Winter } \\
\text { Off Peak }\end{array}$ & $\begin{array}{l}? \\
? \\
?\end{array}$ & $\begin{array}{l}? \\
? \\
?\end{array}$ & $\begin{array}{l}? \\
? \\
?\end{array}$ & $\begin{array}{l}? \\
? \\
?\end{array}$ & $\begin{array}{l}? \\
? \\
?\end{array}$ & By Request & $\begin{array}{l}? \\
? \\
?\end{array}$ & ? & ? \\
\hline $\begin{array}{l}\text { MLA } \\
\text { Summer } \\
\text { Winter } \\
\text { Off Peak }\end{array}$ & $\begin{array}{l}\mathrm{Y} \\
\mathrm{Y} \\
\mathrm{Y}\end{array}$ & $\begin{array}{l}\mathrm{Y} \\
\mathrm{Y} \\
\mathrm{Y}\end{array}$ & $\begin{array}{l}\mathrm{N} \\
\mathrm{N} \\
\mathrm{N}\end{array}$ & $\begin{array}{l}\mathrm{Y} \\
\mathrm{Y} \\
\mathrm{Y}\end{array}$ & $\begin{array}{l}\mathrm{Y} \\
\mathrm{Y} \\
\mathrm{Y} \\
\end{array}$ & $\begin{array}{l}\text { Passenger Service } \\
\text { Pharge } \\
\text { Passenger Service Charge } \\
\text { Passenger Service Charge }\end{array}$ & $\begin{array}{l}3 \text { years staggered } \\
3 \text { years staggered } \\
3 \text { years staggered } \\
\end{array}$ & $\begin{array}{l}\text { new routes -destinations not been served in the last } 12 \text { months, if same airline } 24 \\
\text { months, } 150 \mathrm{~km} \text { away from served airports, } 90 \% \text { scheduled flights operated, } 3 \text { freq summer } \\
2 \text { ffreq winter per week }\end{array}$ & separate incentive for transfer traffic \\
\hline $\begin{array}{l}\text { FUE } \\
\text { Summer } \\
\text { Winter } \\
\text { Off Peak }\end{array}$ & $\begin{array}{l}\mathrm{N} \\
\mathrm{N} \\
\mathrm{Y}\end{array}$ & $\begin{array}{l}\mathrm{Y} \\
\mathrm{Y} \\
\mathrm{Y}\end{array}$ & $\begin{array}{l}\mathrm{N} \\
\mathrm{N} \\
\mathrm{N}\end{array}$ & $\begin{array}{l}\mathrm{Y} \\
\mathrm{Y} \\
\mathrm{Y}\end{array}$ & $\begin{array}{l}\mathrm{Y} \\
\mathrm{Y} \\
\mathrm{Y}\end{array}$ & $\begin{array}{l}\text { Different fees if destination is within EU, Domestic or } \\
\text { Inter-slands }\end{array}$ & Constant & Rate is discounted depending on destination "band" & N/A \\
\hline $\begin{array}{l}\text { TRN } \\
\text { Summer } \\
\text { Winter } \\
\text { Off Peak }\end{array}$ & $\begin{array}{l}\mathrm{N} \\
\mathrm{N} \\
\mathrm{Y}\end{array}$ & $\begin{array}{l}\mathrm{Y} \\
\mathrm{Y} \\
\mathrm{Y}\end{array}$ & $\begin{array}{l}\mathrm{N} \\
\mathrm{N} \\
\mathrm{N}\end{array}$ & $\begin{array}{l}\mathrm{Y} \\
\mathrm{Y} \\
\mathrm{Y}\end{array}$ & $\begin{array}{l}\mathrm{Y} \\
\mathrm{Y} \\
\mathrm{Y}\end{array}$ & Different fees if destination is "EU" or "Extra EU" & Constant & Different fees if destination is "EU" or "Extra EU" & $\mathrm{NA}$ \\
\hline $\begin{array}{l}\text { BOD } \\
\text { Summer } \\
\text { Winter } \\
\text { Off Peak }\end{array}$ & $\begin{array}{l}\mathrm{Y} \\
\mathrm{Y} \\
\mathrm{Y}\end{array}$ & $\begin{array}{l}\mathrm{N} \\
\mathrm{N} \\
\mathrm{N}\end{array}$ & $\begin{array}{l}\mathrm{Y} \\
\mathrm{Y} \\
\mathrm{Y}\end{array}$ & $\begin{array}{l}\mathrm{Y} \\
\mathrm{Y} \\
\mathrm{Y}\end{array}$ & $\begin{array}{l}\mathrm{Y} \\
\mathrm{Y} \\
\mathrm{Y}\end{array}$ & $\begin{array}{l}\text { Landing } \\
\text { Landing Charge } \\
\text { Landing Charge }\end{array}$ & $\begin{array}{l}12 \text { to } 36 \text { month staggered } \\
12 \text { to } 36 \text { month staggered } \\
12 \text { to } 36 \text { month staggered }\end{array}$ & $\begin{array}{l}\text { 50km away from already served market, 40mins car drive away from already served } \\
\text { market, Direct flights, one weekly, two months operation }\end{array}$ & $\mathrm{NA}$ \\
\hline $\begin{array}{l}\text { AES } \\
\text { Summer } \\
\text { Winter } \\
\text { Off Peak }\end{array}$ & $\begin{array}{l}\text { NA } \\
\text { NA } \\
\text { NA }\end{array}$ & $\begin{array}{l}\mathrm{NA} \\
\mathrm{NA} \\
\mathrm{NA}\end{array}$ & $\begin{array}{l}\mathrm{NA} \\
\mathrm{NA} \\
\mathrm{NA}\end{array}$ & $\begin{array}{l}\text { NA } \\
\text { NA } \\
\text { NA }\end{array}$ & $\begin{array}{l}\text { NA } \\
\text { NA } \\
\text { NA }\end{array}$ & Weekly season card & $\begin{array}{l}\mathrm{NA} \\
\mathrm{NA} \\
\mathrm{NA}\end{array}$ & Non commercial, aircraft weight between 1501 - 2000kg & \\
\hline
\end{tabular}


Continued...

\begin{tabular}{|c|c|c|c|c|c|c|c|c|c|}
\hline $\begin{array}{l}\text { BIO } \\
\text { Summer } \\
\text { Winter } \\
\text { Off Peak } \\
\end{array}$ & $\begin{array}{l}\mathrm{N} \\
\mathrm{N} \\
\mathrm{Y} \\
\end{array}$ & $\begin{array}{l}\text { Y } \\
Y \\
Y \\
\end{array}$ & $\begin{array}{l}\mathrm{N} \\
\mathrm{N} \\
\mathrm{N} \\
\end{array}$ & $\begin{array}{l}\mathrm{Y} \\
\mathrm{Y} \\
\mathrm{Y} \\
\end{array}$ & $\begin{array}{l}\mathrm{Y} \\
\mathrm{Y} \\
\mathrm{Y} \\
\end{array}$ & $\begin{array}{l}\text { Different fees if destination is within EU, Domestic or } \\
\text { Inter-slands }\end{array}$ & Constant & Rate is discounted depending on destination "band" & N/A \\
\hline $\begin{array}{l}\text { HAU } \\
\text { Summer } \\
\text { Winter } \\
\text { Off Peak }\end{array}$ & $\begin{array}{l}\text { NA } \\
\text { NA } \\
\text { NA }\end{array}$ & $\begin{array}{l}\mathrm{NA} \\
\mathrm{NA} \\
\mathrm{NA}\end{array}$ & $\begin{array}{l}\mathrm{NA} \\
\mathrm{NA} \\
\mathrm{NA}\end{array}$ & $\begin{array}{l}\text { NA } \\
\text { NA } \\
\text { NA }\end{array}$ & $\begin{array}{l}\mathrm{NA} \\
\mathrm{NA} \\
\mathrm{NA}\end{array}$ & Weekly season card & $\begin{array}{l}\text { NA } \\
\text { NA } \\
\text { NA }\end{array}$ & Non commercial, aircraft weight between 1501 - $2000 \mathrm{~kg}$ & NA \\
\hline $\begin{array}{l}\text { BGO } \\
\text { Summer } \\
\text { Winter } \\
\text { off Peak }\end{array}$ & $\begin{array}{l}\text { NA } \\
\text { NA } \\
\text { NA }\end{array}$ & $\begin{array}{l}\mathrm{NA} \\
\mathrm{NA} \\
\mathrm{NA} \\
\end{array}$ & $\begin{array}{l}\text { NA } \\
\text { NA } \\
\text { NA } \\
\end{array}$ & $\begin{array}{l}\mathrm{NA} \\
\mathrm{NA} \\
\mathrm{NA}\end{array}$ & $\begin{array}{l}\mathrm{NA} \\
\mathrm{NA} \\
\mathrm{NA} \\
\end{array}$ & Weekly season card & $\begin{array}{l}\mathrm{NA} \\
\mathrm{NA} \\
\mathrm{NA}\end{array}$ & Non commercial, aircraft weight between 1501 - 2000kg & NA \\
\hline $\begin{array}{l}\text { MMX } \\
\text { Summer } \\
\text { Winter } \\
\text { Off Peak } \\
\end{array}$ & $\begin{array}{l} \\
Y \\
Y \\
? \\
\end{array}$ & $\begin{array}{l} \\
Y \\
Y \\
Y \\
\end{array}$ & $\begin{array}{l}\mathrm{N} \\
\mathrm{N} \\
\mathrm{N} \\
\end{array}$ & $\begin{array}{l}? \\
? \\
? \\
?\end{array}$ & $\begin{array}{l}? \\
? \\
? \\
?\end{array}$ & Certain fees - must ask for details & ? & New destinations that have not been served during the last 12 months & ? \\
\hline $\begin{array}{l}\text { BOO } \\
\text { Summer } \\
\text { Winter } \\
\text { Off Peak }\end{array}$ & $\begin{array}{l}\mathrm{NA} \\
\mathrm{NA} \\
\mathrm{NA} \\
\end{array}$ & $\begin{array}{l}\mathrm{NA} \\
\mathrm{NA} \\
\mathrm{NA} \\
\end{array}$ & $\begin{array}{l}\mathrm{NA} \\
\mathrm{NA} \\
\mathrm{NA} \\
\end{array}$ & $\begin{array}{l}\mathrm{NA} \\
\mathrm{NA} \\
\mathrm{NA} \\
\end{array}$ & $\begin{array}{l}\mathrm{NA} \\
\mathrm{NA} \\
\mathrm{NA} \\
\end{array}$ & Weekly season card & $\begin{array}{l}\mathrm{NA} \\
\mathrm{NA} \\
\mathrm{NA} \\
\end{array}$ & Non commercial, aircraft weight between 1501 - 2000kg & NA \\
\hline $\begin{array}{l}\text { TRD } \\
\text { Summer } \\
\text { Winter } \\
\text { Off Peak } \\
\end{array}$ & $\begin{array}{l}\mathrm{NA} \\
\mathrm{NA} \\
\mathrm{NA} \\
\end{array}$ & $\begin{array}{l}\mathrm{NA} \\
\mathrm{NA} \\
\mathrm{NA} \\
\end{array}$ & $\begin{array}{l} \\
\text { NA } \\
\text { NA } \\
\text { NA } \\
\end{array}$ & $\begin{array}{l}\mathrm{NA} \\
\mathrm{NA} \\
\mathrm{NA}\end{array}$ & $\begin{array}{l}\mathrm{NA} \\
\mathrm{NA} \\
\mathrm{NA} \\
\end{array}$ & Weekly season card & $\begin{array}{l}\mathrm{NA} \\
\mathrm{NA} \\
\mathrm{NA} \\
\end{array}$ & Non commercial, aircraft weight between 1501 - 2000kg & NA \\
\hline $\begin{array}{l}\text { TOS } \\
\text { Summer } \\
\text { Winter } \\
\text { Off Peak }\end{array}$ & $\begin{array}{l}\mathrm{NA} \\
\mathrm{NA} \\
\mathrm{NA}\end{array}$ & $\begin{array}{l}\mathrm{NA} \\
\mathrm{NA} \\
\mathrm{NA} \\
\end{array}$ & $\begin{array}{l} \\
\text { NA } \\
\text { NA } \\
\text { NA }\end{array}$ & $\begin{array}{l} \\
\mathrm{NA} \\
\mathrm{NA} \\
\mathrm{NA}\end{array}$ & $\begin{array}{l}\mathrm{NA} \\
\mathrm{NA} \\
\mathrm{NA}\end{array}$ & Weekly season card & $\begin{array}{l}\mathrm{NA} \\
\mathrm{NA} \\
\mathrm{NA} \\
\end{array}$ & Non commercial, aircraft weight between 1501 - 2000kg & NA \\
\hline $\begin{array}{l}\text { SVG } \\
\text { Summer } \\
\text { Winter } \\
\text { off Peak }\end{array}$ & $\begin{array}{l}\mathrm{NA} \\
\mathrm{NA} \\
\mathrm{NA} \\
\end{array}$ & $\begin{array}{l}\mathrm{NA} \\
\mathrm{NA} \\
\mathrm{NA} \\
\end{array}$ & $\begin{array}{l}\text { NA } \\
\text { NA } \\
\text { NA } \\
\end{array}$ & $\begin{array}{l}\mathrm{NA} \\
\mathrm{NA} \\
\mathrm{NA}\end{array}$ & $\begin{array}{l}\mathrm{NA} \\
\mathrm{NA} \\
\mathrm{NA} \\
\end{array}$ & Weekly season card & $\begin{array}{l}\mathrm{NA} \\
\mathrm{NA} \\
\mathrm{NA} \\
\end{array}$ & Non commercial, aircraft weight between 1501 - $2000 \mathrm{~kg}$ & NA \\
\hline $\begin{array}{l}\text { TFN } \\
\text { Summer } \\
\text { Winter } \\
\text { Off Peak }\end{array}$ & $\begin{array}{l}\mathrm{N} \\
\mathrm{N} \\
\mathrm{Y}\end{array}$ & $\begin{array}{l}Y \\
Y \\
Y\end{array}$ & $\begin{array}{l}\mathrm{N} \\
\mathrm{N} \\
\mathrm{N}\end{array}$ & $\begin{array}{l}\text { Y } \\
\text { Y } \\
Y\end{array}$ & $\begin{array}{l}\mathrm{Y} \\
\mathrm{Y} \\
\mathrm{Y}\end{array}$ & $\begin{array}{l}\text { Different fees if destination is within EU, Domestic or } \\
\text { Inter-slands }\end{array}$ & Constant & Rate is discounted depending on destination "band" & N/A \\
\hline $\begin{array}{l}\text { KRS } \\
\text { Summer } \\
\text { Winter } \\
\text { Off Peak }\end{array}$ & $\begin{array}{l}\mathrm{NA} \\
\mathrm{NA} \\
\mathrm{NA}\end{array}$ & $\begin{array}{l}\mathrm{NA} \\
\mathrm{NA} \\
\mathrm{NA}\end{array}$ & $\begin{array}{l}\text { NA } \\
\text { NA } \\
\text { NA }\end{array}$ & $\begin{array}{l}\text { NA } \\
\text { NA } \\
\text { NA }\end{array}$ & $\begin{array}{l}\mathrm{NA} \\
\mathrm{NA} \\
\mathrm{NA}\end{array}$ & Weekly season card & $\begin{array}{l}\mathrm{NA} \\
\mathrm{NA} \\
\mathrm{NA}\end{array}$ & Non commercial, aircraft weight between 1501 - $2000 \mathrm{~kg}$ & NA \\
\hline $\begin{array}{l}\mathrm{ABZ} \\
\text { Summer } \\
\text { Winter } \\
\text { Off Peak }\end{array}$ & $\begin{array}{l}\text { Apply } \\
\text { Apply } \\
\text { Apply }\end{array}$ & $\begin{array}{l}\text { Y } \\
Y \\
Y \\
\end{array}$ & $\begin{array}{l}\text { Apply } \\
\text { Apply } \\
\text { Apply }\end{array}$ & $\begin{array}{l}\mathrm{Y} \\
\mathrm{Y} \\
\mathrm{Y} \\
\end{array}$ & $\begin{array}{l} \\
Y \\
Y \\
Y \\
\end{array}$ & Weight \& Pax charge depending & Constant & $\begin{array}{l}\text { Flights within Scotland 65\% weight discount, flights < } 250 \text { statue miles weight } \\
26 \% \text { discount, } \\
\text { Fixed wing \& destination is within } 100 \text { statute miles } 50 \% \text { PAX charge rebate } \\
\text { Flight originating in UK landing to pickup PAX / cargo 50\% weight discount }\end{array}$ & Training flights $70 \%$ off total charge calculated. \\
\hline $\begin{array}{l}\text { HHN } \\
\text { Summer } \\
\text { Winter } \\
\text { Off Peak }\end{array}$ & $\begin{array}{l}\mathrm{NA} \\
\mathrm{NA} \\
\mathrm{NA}\end{array}$ & $\begin{array}{l}\mathrm{NA} \\
\mathrm{NA} \\
\mathrm{NA}\end{array}$ & $\begin{array}{l}\text { NA } \\
\text { NA } \\
\text { NA }\end{array}$ & $\begin{array}{l}\text { NA } \\
\text { NA } \\
\text { NA }\end{array}$ & $\begin{array}{l}\mathrm{NA} \\
\mathrm{NA} \\
\mathrm{NA}\end{array}$ & None Stated & NA & NA & NA \\
\hline $\begin{array}{l}\text { LGG } \\
\text { Summer } \\
\text { Winter } \\
\text { Off Peak }\end{array}$ & $\begin{array}{l}\mathrm{NA} \\
\mathrm{NA} \\
\mathrm{NA} \\
\end{array}$ & $\begin{array}{l}\mathrm{NA} \\
\mathrm{NA} \\
\mathrm{NA} \\
\end{array}$ & $\begin{array}{l}\mathrm{NA} \\
\mathrm{NA} \\
\mathrm{NA} \\
\end{array}$ & $\begin{array}{l}\text { NA } \\
\text { NA } \\
\text { NA }\end{array}$ & $\begin{array}{l}\mathrm{NA} \\
\mathrm{NA} \\
\mathrm{NA} \\
\end{array}$ & None Stated & NA & NA & NA \\
\hline
\end{tabular}


Continued...

\begin{tabular}{|c|c|c|c|c|c|c|c|c|c|}
\hline & \begin{tabular}{|l} 
New Destinations \\
\end{tabular} & Specific Target & Increased Feq. & Off Peak & On Peak & Item Discounted & Duration & $\begin{array}{l}\text { Min. Requirements } \\
\end{array}$ & $\begin{array}{ll}\text { Special Offer } \\
\end{array}$ \\
\hline $\begin{array}{l}\text { AMS } \\
\text { Summer } \\
\text { Winter } \\
\text { off Peak }\end{array}$ & $\begin{array}{l}\mathrm{Y} \\
\mathrm{Y} \\
\mathrm{Y}\end{array}$ & $\begin{array}{l}\mathrm{N} \\
\mathrm{N} \\
\mathrm{N}\end{array}$ & 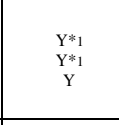 & $\begin{array}{c}\mathrm{Y} \\
\mathrm{Y} \\
\mathrm{Y}\end{array}$ & $\begin{array}{l}\mathrm{Y} \\
\mathrm{Y} \\
\mathrm{N}\end{array}$ & Reward per departing PAX & $\begin{array}{l}3 \text { years staggered } \\
3 \text { years staggered } \\
1 \text { year }\end{array}$ & $\begin{array}{l}3 \mathrm{x} \text { weekly, } 20 \text { weeks } 1 \text { weekly*1 } \\
\text { Increase number of flights, must operate min } 80 \% \text { of flights, } 1 \text { weekly } 20 \text { weeks }\end{array}$ & Seat capacity increase eligible ${ }^{*_{1}}$ Start up airline bonus ${ }^{*}{ }_{1}$ \\
\hline $\begin{array}{l}\text { ATH } \\
\text { Summer } \\
\text { Winter } \\
\text { off Peak }\end{array}$ & $\begin{array}{l}\mathrm{N} \\
\mathrm{N} \\
\mathrm{N}\end{array}$ & $\begin{array}{l}\mathrm{N} \\
\mathrm{N} \\
\mathrm{N}\end{array}$ & $\begin{array}{l}\mathrm{N} \\
\mathrm{N} \\
\mathrm{N}\end{array}$ & $\begin{array}{l}\mathrm{Y} \\
\mathrm{Y} \\
\mathrm{Y}\end{array}$ & $\begin{array}{l}\mathrm{N} \\
\mathrm{N} \\
\mathrm{N}\end{array}$ & $\begin{array}{l}\text { Parking } \\
\text { PParing } \\
\text { Parking }\end{array}$ & $\begin{array}{l}\text { on-going } \\
\text { on-going } \\
\text { on-going }\end{array}$ & Parking between 24:00 \& 05:00 & $\mathrm{N} / \mathrm{A}$ \\
\hline $\begin{array}{l}\text { BHX } \\
\text { Summer } \\
\text { Winter } \\
\text { off Peak }\end{array}$ & $\begin{array}{c}\mathrm{Y} \\
\mathrm{Y} \\
\mathrm{Y}\end{array}$ & $\begin{array}{l}\mathrm{N} \\
\mathrm{N} \\
\mathrm{N}\end{array}$ & $\begin{array}{l}\mathrm{N} \\
\mathrm{N} \\
\mathrm{N}\end{array}$ & $\begin{aligned} \mathrm{Y} \\
\mathrm{Y} \\
\mathrm{Y}\end{aligned}$ & $\begin{array}{c}\mathrm{Y} \\
\mathrm{Y} \\
\mathrm{Y}\end{array}$ & 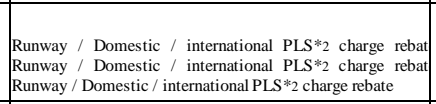 & $\begin{array}{l}4 \text { years staggered } \\
4 \text { years staggered } \\
4 \text { years staggered }\end{array}$ & Scheduled airline, Growth ${ }^{*}$ in subsequent years, ICAO chap. 3 aircraft & Alternative scheme for tour operators \\
\hline $\begin{array}{l}\text { BMA } \\
\text { Summer } \\
\text { Winter } \\
\text { Off Peak }\end{array}$ & $\begin{array}{l}\mathrm{Y} \\
\mathrm{Y} \\
?\end{array}$ & $\begin{array}{l}\mathrm{Y} \\
\mathrm{Y} \\
\mathrm{Y}\end{array}$ & $\begin{array}{l}\mathrm{N} \\
\mathrm{N} \\
\mathrm{N}\end{array}$ & $\stackrel{?}{?} ?$ & $\begin{array}{l}? \\
? \\
?\end{array}$ & Certain fees - must ask for details & ? & New destinations that have not been served during the last 12 months & ? \\
\hline $\begin{array}{l}\text { BRU } \\
\text { Summer } \\
\text { Winter } \\
\text { Off Peak } \\
\end{array}$ & $\begin{array}{l}\mathrm{Y} \\
\mathrm{Y} \\
\mathrm{Y} \\
\end{array}$ & $\begin{array}{l}\mathrm{N} \\
\mathrm{N} \\
\mathrm{N} \\
\end{array}$ & $\begin{array}{l}\mathrm{Y}^{*_{1}} \\
\mathrm{Y}^{*}{ }^{*} \\
\mathrm{Y}^{*}{ }_{1} \\
\end{array}$ & $\begin{array}{l}\mathrm{Y} \\
\mathrm{Y} \\
\mathrm{Y}\end{array}$ & $\begin{array}{l}\mathrm{Y} \\
\mathrm{Y} \\
\mathrm{Y} \\
\end{array}$ & $\begin{array}{l}\text { Passenger service charge } \\
\text { Passenger sevice charge } \\
\text { Passenger service charge }\end{array}$ & $\begin{array}{l}3 \text { years staggered } \\
3 \text { years staggered } \\
3 \text { years staggered } \\
\end{array}$ & None stated & Alternative scheme for cargo \\
\hline $\begin{array}{l}\text { FRA } \\
\text { Summer } \\
\text { Winter } \\
\text { Off Peak }\end{array}$ & $\begin{array}{l}\mathrm{N} \\
\mathrm{N} \\
\mathrm{N}\end{array}$ & $\begin{array}{l}\mathrm{N} \\
\mathrm{N} \\
\mathrm{N}\end{array}$ & $\begin{array}{l}\mathrm{N} \\
\mathrm{N} \\
\mathrm{N}\end{array}$ & $\begin{array}{l}\mathrm{Y} \\
\mathrm{Y} \\
\mathrm{Y}\end{array}$ & $\begin{array}{l}\mathrm{Y} \\
\mathrm{Y} \\
\mathrm{Y}\end{array}$ & $\begin{array}{l}\text { Passenger Fee Cap } \\
\text { Passenger Fee Cap } \\
\text { Passenger Fee Cap }\end{array}$ & $\begin{array}{l}\text { on-going } \\
\text { on-going } \\
\text { on-going }\end{array}$ & SLF $>83.0 \% \& 89.9 \%, 90.0 \% \& 96.9 \%,>97.0 \%$, min 150 take-offs with PAX on board & N/A \\
\hline $\begin{array}{l}\text { GOT } \\
\text { Summer } \\
\text { Winter } \\
\text { Off Peak }\end{array}$ & $\stackrel{?}{?} ?$ & $\begin{array}{l}? \\
? \\
?\end{array}$ & $\stackrel{?}{?} ?$ & $\stackrel{?}{?} ?$ & $\begin{array}{l}? \\
? \\
?\end{array}$ & $\stackrel{?}{?} ?$ & $\begin{array}{l}? \\
? \\
?\end{array}$ & new routes to destinations that have not been served during the last 12 months & \\
\hline $\begin{array}{l}\text { LIS } \\
\text { Summer } \\
\text { Winter } \\
\text { Off Peak } \\
\end{array}$ & $\begin{array}{l}\mathrm{NA} \\
\mathrm{NA} \\
\mathrm{NA}\end{array}$ & $\begin{array}{l}\mathrm{NA} \\
\mathrm{NA} \\
\mathrm{NA}\end{array}$ & $\begin{array}{l}\mathrm{NA} \\
\mathrm{NA} \\
\mathrm{NA}\end{array}$ & $\begin{array}{l}\mathrm{NA} \\
\mathrm{NA} \\
\mathrm{NA}\end{array}$ & $\begin{array}{l}\text { NA } \\
\text { NA } \\
\text { NA }\end{array}$ & None Stated & $\mathrm{NA}$ & $\mathrm{NA}$ & $\mathrm{NA}$ \\
\hline $\begin{array}{l}\text { LGW } \\
\text { Summer } \\
\text { Winter } \\
\text { Off Peak } \\
\end{array}$ & $\begin{array}{l}\mathrm{NA} \\
\mathrm{NA} \\
\mathrm{NA}\end{array}$ & $\begin{array}{l}\mathrm{NA} \\
\mathrm{NA} \\
\mathrm{NA}\end{array}$ & $\begin{array}{l}\mathrm{NA} \\
\mathrm{NA} \\
\mathrm{NA} \\
\end{array}$ & $\begin{array}{l}\mathrm{NA} \\
\mathrm{NA} \\
\mathrm{NA} \\
\end{array}$ & $\begin{array}{l}\mathrm{NA} \\
\mathrm{NA} \\
\mathrm{NA}\end{array}$ & None Stated & $\mathrm{NA}$ & $\mathrm{NA}$ & $\mathrm{NA}$ \\
\hline $\begin{array}{l}\text { MAN } \\
\text { Summer } \\
\text { Winter } \\
\text { off Peak }\end{array}$ & $?$ & $?$ & $?$ & $\begin{array}{l}? \\
? \\
?\end{array}$ & $?$ & $?$ & $?$ & 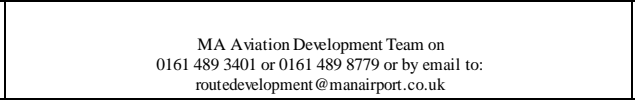 & \\
\hline 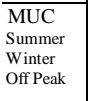 & $\begin{array}{l}\mathrm{NA} \\
\mathrm{NA} \\
\mathrm{NA}\end{array}$ & $\begin{array}{l}\mathrm{NA} \\
\text { NA } \\
\text { NA }\end{array}$ & $\begin{array}{l}\mathrm{NA} \\
\mathrm{NA} \\
\mathrm{NA}\end{array}$ & $\begin{array}{l}\mathrm{NA} \\
\mathrm{NA} \\
\mathrm{NA}\end{array}$ & $\begin{array}{l}\mathrm{NA} \\
\mathrm{NA} \\
\mathrm{NA}\end{array}$ & No discount, but surcharge for polluting aircraft & NA & $\mathrm{NA}$ & \\
\hline $\begin{array}{l}\text { NCE } \\
\text { Summer } \\
\text { Winter } \\
\text { Off Peak } \\
\end{array}$ & $\begin{array}{l}\mathrm{Y} \\
\mathrm{Y} \\
\mathrm{Y} \\
\end{array}$ & $\begin{array}{l}\mathrm{N} \\
\mathrm{N} \\
\mathrm{N} \\
\end{array}$ & $\begin{array}{l}\mathrm{Y} \\
\mathrm{Y} \\
\mathrm{Y} \\
\end{array}$ & $\begin{array}{l}\mathrm{Y} \\
\mathrm{Y} \\
\mathrm{Y}\end{array}$ & $\begin{array}{l}\mathrm{Y} \\
\mathrm{Y} \\
\mathrm{Y} \\
\end{array}$ & $\begin{array}{l}\text { Landing, Passenger bonus if grownth over } 4.7 \% \\
\text { Landing, P.sasengererbous sif ifrowht over } 3 \% \\
\text { Landing, Passenger }\end{array}$ & $\begin{array}{l}2 \text { years staggered } \\
2 \text { years staggered } \\
2 \text { years staggered } \\
\end{array}$ & $\begin{array}{l}\text { new routes -destinations not been served in the last } 12 \text { months, } 24 \text { freq summer } \\
16 \text { freq winter }\end{array}$ & Extra bonus discount for new long haul traffic for 3 years \\
\hline $\begin{array}{l}\text { PRG } \\
\text { Summer } \\
\text { Winter } \\
\text { Off Peak } \\
\end{array}$ & $\begin{array}{l}\mathrm{Y} \\
\mathrm{Y} \\
\mathrm{N}\end{array}$ & $\begin{array}{l}\mathrm{Y} \\
\mathrm{Y} \\
\mathrm{N}\end{array}$ & $\begin{array}{l}\mathrm{N} \\
\mathrm{N} \\
\mathrm{N}\end{array}$ & $\begin{array}{l}\mathrm{Y} \\
\mathrm{Y} \\
\mathrm{Y} \\
\end{array}$ & $\begin{array}{l}\mathrm{Y} \\
\mathrm{Y} \\
\mathrm{N}\end{array}$ & $\begin{array}{c}\text { Landing fees } \\
\text { Landing gess } \\
50 \% \text { off parking fees }\end{array}$ & $\begin{array}{l}3 \text { years staggered } \\
3 \text { years staggered } \\
\text { on-going }\end{array}$ & $\begin{array}{l}\text { IATA geographic area TC2 and named, } 1 \text { weekly } \\
07: 30-09: 30 \text { and 12:35 - 14:35 local }\end{array}$ & increase capacity on routes served for $>12$ months, MTOW $>100$, use of air bridge \\
\hline
\end{tabular}




\begin{tabular}{|c|c|c|c|c|c|c|c|c|c|}
\hline & & & & & & & \multicolumn{3}{|c|}{$1 \ldots$} \\
\hline $\begin{array}{l}\text { BRI } \\
\text { Summer } \\
\text { Winter } \\
\text { Off Peak }\end{array}$ & $\begin{array}{l}\mathrm{N} \\
\mathrm{N} \\
\mathrm{N}\end{array}$ & $\begin{array}{l}\mathrm{Y} \\
\mathrm{Y} \\
\mathrm{Y}\end{array}$ & $\begin{array}{l}\mathrm{N} \\
\mathrm{N} \\
\mathrm{N}\end{array}$ & $\begin{array}{l}\mathrm{Y} \\
\mathrm{Y} \\
\mathrm{Y}\end{array}$ & $\begin{array}{l}\mathrm{Y} \\
\mathrm{Y} \\
\mathrm{Y}\end{array}$ & Different fees if destination is International or Domestic & Constant & Depending if international or domestic & $\mathrm{N} / \mathrm{A}$ \\
\hline $\begin{array}{l}\text { NAP } \\
\text { Summer } \\
\text { Winter } \\
\text { Off Peak }\end{array}$ & $\begin{array}{l}\mathrm{N} \\
\mathrm{N} \\
\mathrm{N}\end{array}$ & $\begin{array}{l}\mathrm{Y} \\
\mathrm{Y} \\
\mathrm{Y}\end{array}$ & $\begin{array}{l}\mathrm{N} \\
\mathrm{N} \\
\mathrm{N}\end{array}$ & $\begin{array}{l}\mathrm{Y} \\
\mathrm{Y} \\
\mathrm{Y}\end{array}$ & $\begin{array}{l}\mathrm{Y} \\
\mathrm{Y} \\
\mathrm{Y}\end{array}$ & Different fees if destination is International or Domestic & Constant & Depending if international or domestic & $\mathrm{N} / \mathrm{A}$ \\
\hline $\begin{array}{l}\text { VLC } \\
\text { Summer } \\
\text { Winter } \\
\text { Off Peak }\end{array}$ & $\begin{array}{l}\mathrm{N} \\
\mathrm{N} \\
\mathrm{Y}\end{array}$ & $\begin{array}{l}\mathrm{Y} \\
\mathrm{Y} \\
\mathrm{Y}\end{array}$ & $\begin{array}{l}\mathrm{N} \\
\mathrm{N} \\
\mathrm{N}\end{array}$ & $\begin{array}{l}\mathrm{Y} \\
\mathrm{Y} \\
\mathrm{Y}\end{array}$ & $\begin{array}{l}\mathrm{Y} \\
\mathrm{Y} \\
\mathrm{Y}\end{array}$ & $\begin{array}{l}\text { Different fees if destination is within EU, Domestic or } \\
\text { Inter-slslands }\end{array}$ & Constant & Rate is discounted depending on destination "band" & N/A \\
\hline $\begin{array}{l}\text { AGP } \\
\text { Summer } \\
\text { Winter } \\
\text { Off Peak }\end{array}$ & $\begin{array}{l}\mathrm{N} \\
\mathrm{N} \\
\mathrm{Y}\end{array}$ & $\begin{array}{l}\mathrm{Y} \\
\mathrm{Y} \\
\mathrm{Y}\end{array}$ & $\begin{array}{l}\mathrm{N} \\
\mathrm{N} \\
\mathrm{N}\end{array}$ & $\begin{array}{c}\mathrm{Y} \\
\mathrm{Y} \\
\mathrm{Y}\end{array}$ & $\begin{array}{l}\mathrm{Y} \\
\mathrm{Y} \\
\mathrm{Y}\end{array}$ & $\begin{array}{l}\text { Different fees if destination is within EU, Domestic or } \\
\text { Inter-slands }\end{array}$ & Constant & Rate is discounted depending on destination "band" & $\mathrm{N} / \mathrm{A}$ \\
\hline $\begin{array}{l}\text { LPA } \\
\text { Summer } \\
\text { Winter } \\
\text { Off Peak }\end{array}$ & $\begin{array}{l}\mathrm{N} \\
\mathrm{N} \\
\mathrm{Y}\end{array}$ & $\begin{array}{l}\mathrm{Y} \\
\mathrm{Y} \\
\mathrm{Y}\end{array}$ & $\begin{array}{l}\mathrm{N} \\
\mathrm{N} \\
\mathrm{N}\end{array}$ & $\begin{array}{l}\mathrm{Y} \\
\mathrm{Y} \\
\mathrm{Y}\end{array}$ & $\begin{array}{l}\mathrm{Y} \\
\mathrm{Y} \\
\mathrm{Y}\end{array}$ & $\begin{array}{l}\text { Different fees if destination is within EU, Domestic or } \\
\text { Inter-slands }\end{array}$ & Constant & Rate is discounted depending on destination "band" & $\mathrm{N} / \mathrm{A}$ \\
\hline $\begin{array}{l}\text { ALC } \\
\text { Summer } \\
\text { Winter } \\
\text { Off Peak }\end{array}$ & $\begin{array}{l}\mathrm{N} \\
\mathrm{N} \\
\mathrm{Y}\end{array}$ & $\begin{array}{l}\mathrm{Y} \\
\mathrm{Y} \\
\mathrm{Y}\end{array}$ & $\begin{array}{l}\mathrm{N} \\
\mathrm{N} \\
\mathrm{N}\end{array}$ & $\begin{array}{l}\mathrm{Y} \\
\mathrm{Y} \\
\mathrm{Y}\end{array}$ & $\begin{array}{l}\mathrm{Y} \\
\mathrm{Y} \\
\mathrm{Y}\end{array}$ & $\begin{array}{l}\text { Different fees if destination is within EU, Domestic or } \\
\text { Inter-rslands }\end{array}$ & Constant & Rate is discounted depending on destination "band" & $\mathrm{N} / \mathrm{A}$ \\
\hline $\begin{array}{l}\text { PMI } \\
\text { Summer } \\
\text { Winter } \\
\text { Off Peak }\end{array}$ & $\begin{array}{l}\mathrm{N} \\
\mathrm{N} \\
\mathrm{Y}\end{array}$ & $\begin{array}{l}\mathrm{Y} \\
\mathrm{Y} \\
\mathrm{Y}\end{array}$ & $\begin{array}{l}\mathrm{N} \\
\mathrm{N} \\
\mathrm{N}\end{array}$ & $\begin{array}{l}\mathrm{Y} \\
\mathrm{Y} \\
\mathrm{Y}\end{array}$ & $\begin{array}{l}\mathrm{Y} \\
\mathrm{Y} \\
\mathrm{Y}\end{array}$ & $\begin{array}{l}\text { Different fees if destination is within EU, Domestic or } \\
\text { Inter-rslands }\end{array}$ & Constant & Rate is discounted depending on destination "band" & $\mathrm{N} / \mathrm{A}$ \\
\hline
\end{tabular}

1 long haul, 2 PLS = PAX load Supplement, 3 growth on tonnage and PAX, Y Feature present, N Feature absent, and ? Not known 
Table 4: Duration of incentive schemes

\begin{tabular}{ll}
\hline Aena controlled airports, NAP, BDS, OPO, TRN, ABZ & No stated review period \\
\hline ATH, FRA & Reviewed by airport management \\
BHX & 4 years \\
AMS, MLA, PRG, BOD & 3 years \\
HAJ & 5 flight plan periods \\
NCE & 2 years \\
AMS (off-peak) PRG (off-peak) & 1 year \\
Avinor AS controlled airports & 1 week \\
GOT, MAN, DBV, MMX, BMA & Unknown \\
RIX, HHN, LGG, MUC, LGW, LIS & N/A \\
\hline
\end{tabular}

Table 5: List of items that are discounted

\begin{tabular}{ll}
\hline Domestic flights & BHX, GRO, NAP, BRI, BDS, (Aena controlled airports) \\
International flights & BHX, GRO, NAP, BRI, BDS, (Aena controlled airports) \\
Inter-island services & (Aena controlled airports) \\
Parking fees & ATH, PRG \\
PAX fees & BHX, BRU, FRA, MLA, NCE \\
Runway / Landing fees & BHX, NCE, PRG, BOD \\
Weight charge & ABZ \\
\hline
\end{tabular}


Table 6: Calculations for Lufthansa A340-300 incentive scheme benchmarking exercise - standard operations

\begin{tabular}{|c|c|c|c|c|c|c|c|c|c|c|c|c|c|c|c|c|c|c|c|c|}
\hline \multirow{2}{*}{$\begin{array}{l}\text { Airport } \\
\text { Year } \\
\text { Runway Charges }\end{array}$} & \multirow{2}{*}{\multicolumn{5}{|c|}{ MLA }} & \multicolumn{5}{|c|}{ NCE } & \multicolumn{5}{|c|}{ BRU } & \multicolumn{5}{|c|}{ BHX } \\
\hline & & & & & & 0 & 1 & & 3 & 4 & 0 & 1 & 2 & 3 & 4 & 0 & 1 & 2 & 3 & 4 \\
\hline & 1010.00 & 1010.00 & 1010.00 & 1010.00 & 1010.00 & 1734.35 & 433.59 & 867.18 & 1734.35 & 1734.35 & 773.50 & 773.50 & 773.50 & 773.50 & 773.50 & 3166.94 & 0.00 & 775.95 & 1551.91 & 2327.86 \\
\hline Enuironment & 0.00 & 0.00 & 0.00 & 0.00 & 0.00 & -252.85 & -57.74 & -122.78 & -252.85 & -252.85 & -116.02 & -116.02 & -116.02 & -116.02 & -116.02 & 0.00 & 0.00 & 0.00 & 0.00 & 0.00 \\
\hline Terminal Nav. & 0.00 & & 0.00 & 0.00 & 0.00 & 903.20 & 903.20 & 903.20 & 903.20 & 903.20 & 548.76 & 548.76 & 548.76 & 548.76 & 548.76 & 848.00 & 831.09 & 831.09 & 831.09 & 831.09 \\
\hline Terminal Charges & & & & & & & & & & & & & & & & & & & & \\
\hline Infrastructure & 0.00 & 0.00 & 0.00 & 0.00 & 0.00 & 0.00 & 0.00 & 0.00 & 0.00 & 0.00 & 5.66 & 5.66 & 5.66 & 5.66 & 5.66 & 0.00 & 0.00 & 0.00 & 0.00 & 0.00 \\
\hline Airbridge & 0.00 & 0.00 & 0.00 & 0.00 & 0.00 & 39.74 & 39.74 & 39.74 & 39.74 & 39.74 & 0.00 & 0.00 & 0.00 & 0.00 & 0.00 & 0.00 & 0.00 & 0.00 & 0.00 & 0.00 \\
\hline Aircraft Parking & 0.00 & 0.00 & 0.00 & 0.00 & 0.00 & 0.00 & 0.00 & 0.00 & 0.00 & 0.00 & 0.00 & 0.00 & 0.00 & 0.00 & 0.00 & 0.00 & 0.00 & 0.00 & 0.00 & 0.00 \\
\hline Airport Charges & 1010.00 & 1010.00 & 1010.00 & 1010.00 & 1010.00 & 2424.00 & 1319.00 & 1687.00 & 2424.00 & 2424.00 & 1212.00 & 1212.00 & 1212.00 & 1212.00 & 1212.00 & 4015.00 & 831.00 & 1607.00 & 2383.00 & 3159.00 \\
\hline Average Per Pax & 5.84 & 5.84 & 5.84 & 5.84 & 5.84 & 14.01 & 7.62 & 9.75 & 14.01 & 14.01 & 7.01 & 7.01 & 7.01 & 7.01 & 7.01 & 23.21 & 4.80 & 9.29 & 13.77 & 18.26 \\
\hline $\begin{array}{l}\text { Pax Charges } \\
\text { Passenger }\end{array}$ & 2899.48 & 2029.29 & 2319.93 & 2608.84 & 2899.48 & 1335.56 & 667.78 & 934.20 & 1335.56 & 1335.56 & 3525.74 & 1762.87 & 2645.17 & 2645.17 & 3525.74 & 2300.90 & 1127.96 & 1420.33 & 1691.94 & 1982.58 \\
\hline Transfer Pax & 0.00 & 0.00 & 0.0 & 0.00 & 0 & 0.00 & 0.00 & 0.00 & 0 & 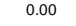 & ( & & & & 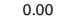 & & .00 & 0.00 & 0.00 & 0.00 \\
\hline $\begin{array}{l}\text { Security } \\
\text { Transfers }\end{array}$ & 378.87 & $\begin{array}{l}378.87 \\
0.00\end{array}$ & $\begin{array}{l}378.87 \\
0.00\end{array}$ & 378.87 & 378.87 & $\begin{array}{l}1818.23 \\
0.00\end{array}$ & 1818.23 & 1818.23 & 1818.23 & 1818.23 & ${ }^{1105.47}$ & 1105.47 & 1105.47 & 1105.47 & 1105.47 & 51.90 & 50.17 & 50.17 & 50.17 & 50.17 \\
\hline $\begin{array}{l}\text { Transfer Security } \\
\text { Infrastructure }\end{array}$ & $\begin{array}{l}0.00 \\
24.22\end{array}$ & $\begin{array}{l}0.00 \\
24.22\end{array}$ & $\begin{array}{l}0.00 \\
24.22\end{array}$ & $\begin{array}{l}0.00 \\
24.22\end{array}$ & $\begin{array}{l}0.00 \\
24.22\end{array}$ & $\begin{array}{l}0.00 \\
0.00\end{array}$ & $\begin{array}{l}0.00 \\
0.00\end{array}$ & $\begin{array}{l}0.00 \\
0.00\end{array}$ & $\begin{array}{l}0.00 \\
0.00\end{array}$ & $\begin{array}{l}0.00 \\
0.00\end{array}$ & $\begin{array}{l}0.00 \\
0.00\end{array}$ & $\begin{array}{l}0.00 \\
0.00\end{array}$ & $\begin{array}{l}0.00 \\
0.00\end{array}$ & $\begin{array}{l}0.00 \\
0.00\end{array}$ & $\begin{array}{l}0.00 \\
0.00\end{array}$ & $\begin{array}{l}0.00 \\
2076\end{array}$ & $\begin{array}{l}0.00 \\
20.76\end{array}$ & $\begin{array}{l}0.00 \\
2076\end{array}$ & $\begin{array}{l}0.00 \\
2076\end{array}$ & 2076 \\
\hline $\begin{array}{l}\text { Intrastrucure } \\
\text { Gou. Taxes }\end{array}$ & $\begin{array}{l}24.22 \\
0.00\end{array}$ & $\begin{array}{l}24.22 \\
0.00\end{array}$ & $\begin{array}{l}24.22 \\
0.00\end{array}$ & $\begin{array}{c}24.22 \\
0.00\end{array}$ & $\begin{array}{l}24.22 \\
0.00\end{array}$ & $\begin{array}{l}0.00 \\
0.00\end{array}$ & $\begin{array}{l}0.00 \\
0.00\end{array}$ & 0.00 & 0.00 & 0.00 & $\begin{array}{l}0.00 \\
0.00\end{array}$ & $\begin{array}{l}0.00 \\
0.00\end{array}$ & $\begin{array}{l}0.00 \\
0.00\end{array}$ & $\begin{array}{l}0.00 \\
0.00\end{array}$ & $\begin{array}{l}0.00 \\
0.00\end{array}$ & $\begin{array}{l}20.16 \\
0.00\end{array}$ & $\begin{array}{l}20.16 \\
0.00\end{array}$ & $\begin{array}{l}20.16 \\
0.00\end{array}$ & $\begin{array}{l}20.16 \\
0.00\end{array}$ & $\begin{array}{c}20.76 \\
0.00\end{array}$ \\
\hline Total Pax Charge & $\begin{array}{r}3303.00 \\
3000\end{array}$ & 2432.00 & 2723.00 & 3012.00 & 3303.00 & 3154.00 & 2486.00 & 2752.00 & 3154.00 & 3154.00 & 4631.00 & 2868.00 & 3751.00 & 3751.00 & 4631.00 & 2374.00 & 1199.00 & 1491.00 & 1763.00 & 2054.00 \\
\hline Average Pax Charge & 19.09 & 14.06 & 15.74 & 17.41 & 19.09 & 18.23 & 14.37 & 15.91 & 18.23 & 18.23 & 26.77 & 16.58 & 21.68 & 21.68 & 26.77 & 13.72 & 6.93 & 8.62 & 10.19 & 11.87 \\
\hline $\begin{array}{l}\text { thal Charges } \\
\text { evaga Per Pax }\end{array}$ & $\begin{array}{l}4313.00 \\
24.93\end{array}$ & $\begin{array}{l}3442.00 \\
19.90\end{array}$ & $\begin{array}{l}3733.00 \\
22.58\end{array}$ & $\begin{array}{l}4022.00 \\
23.25\end{array}$ & $\begin{array}{l}4313.00 \\
24.93\end{array}$ & $\begin{array}{l}5578.00 \\
32.24\end{array}$ & $\begin{array}{l}3805.00 \\
21.99\end{array}$ & $\begin{array}{l}4440.00 \\
25.66\end{array}$ & $\begin{array}{l}5578.00 \\
32.24\end{array}$ & $\begin{array}{c}5578.00 \\
32.24\end{array}$ & $\begin{array}{c}5843.00 \\
33.78\end{array}$ & $\begin{array}{l}4080.00 \\
23.59\end{array}$ & $\begin{array}{l}4963.00 \\
28.69\end{array}$ & $\begin{array}{c}4963.00 \\
28.69\end{array}$ & $\begin{array}{c}5843.00 \\
33.78\end{array}$ & $\begin{array}{c}6389.00 \\
36.93\end{array}$ & $\begin{array}{c}2030.00 \\
11.73\end{array}$ & $\begin{array}{c}3098.00 \\
17.91\end{array}$ & $\begin{array}{c}4146.00 \\
23.96\end{array}$ & $\begin{array}{r}5212.00 \\
2313\end{array}$ \\
\hline
\end{tabular}

\begin{tabular}{|lll|}
\hline Assumptions & Currency: EUR 1.00 EUR=0.83GBP \\
Airline: Luthansa & Mrow: 271 tonnes & Capacity: 247 \\
Aircatt: A340-300 & MLW: 190 tonnes & Load Factor: 70\% \\
Route: intra EU & Passengers: 173 \\
\hline
\end{tabular}


Table 7: Calculations for Lufthansa A340-300 incentive scheme benchmarking exercise strategic operations

\begin{tabular}{|c|c|c|c|c|c|c|c|c|c|c|c|c|c|c|c|c|c|c|c|c|}
\hline \multirow{5}{*}{$\begin{array}{l}\text { Airport } \\
\text { Year } \\
\text { Runway Charges } \\
\text { Landing } \\
\text { Environment } \\
\text { Terminal Nav. }\end{array}$} & \multicolumn{5}{|c|}{ MLA } & \multicolumn{5}{|c|}{ NCE } & \multicolumn{5}{|c|}{ BRU } & \multicolumn{5}{|c|}{ BHX } \\
\hline & 0 & 1 & 2 & 3 & 4 & 0 & 1 & 2 & 3 & 4 & 0 & 1 & 2 & 3 & 4 & 0 & 1 & 2 & 3 & 4 \\
\hline & 1010.00 & 1010.00 & 1010.00 & 1010.00 & 1010.00 & 1734.35 & 433.59 & 867.18 & 1040.61 & 1734.35 & 773.50 & 773.50 & 773.50 & 773.50 & 773.50 & 3166.94 & 0.00 & 775.95 & 1551.91 & 2327.86 \\
\hline & 0.00 & 0.00 & 0.00 & 0.000 & 0.00 & -252.85 & -57.74 & -122.78 & -148.79 & $\begin{array}{l}-252.85 \\
\text { - }\end{array}$ & -116.02 & -116.02 & -116.02 & -116.02 & -116.02 & 0.00 & 0.00 & 0.00 & 0.00 & $\begin{array}{c}32.7 .80 \\
0.00\end{array}$ \\
\hline & 0.00 & 0.00 & 0.00 & 0.00 & 0.00 & 903.20 & 903.20 & 903.20 & 903.20 & 903.20 & 548.76 & 548.76 & 548.76 & 548.76 & 548.76 & 848.00 & 831.09 & 831.09 & 831.09 & 831.09 \\
\hline \multicolumn{21}{|l|}{ Terminal Charges } \\
\hline Infrastructure & 0.00 & 0.00 & 0.00 & 0.00 & 0.00 & 0.00 & 0.00 & 0.00 & 0.00 & 0.00 & 5.66 & 5.66 & 5.66 & 5.66 & 5.66 & 0.00 & 0.00 & 0.00 & 0.00 & 0.00 \\
\hline Airbridge & 0.00 & 0.00 & 0.00 & 0.00 & 0.00 & 39.74 & 39.74 & 39.74 & 39.74 & 39.74 & 0.00 & 0.00 & 0.00 & 0.00 & 0.00 & 0.00 & 0.00 & 0.00 & 0.00 & 0.00 \\
\hline Aircraftt Parking & 0.00 & 0.00 & 0.00 & 0.00 & 0.00 & 0.00 & 0.00 & 0.00 & 0.00 & 0.00 & 0.00 & 0.00 & 0.00 & 0.00 & 0.00 & 0.00 & 0.00 & 0.00 & 0.00 & 0.00 \\
\hline Airport Charges & 1010.00 & 1010.00 & 1010.00 & 1010.00 & 1010.00 & 2424.00 & 1319.00 & 1687.00 & 1835.00 & 2424.00 & 1212.00 & 1212.00 & 1212.00 & 1212.00 & 1212.00 & 4015.00 & 831.00 & 1607.00 & 2383.00 & 3159.00 \\
\hline Average Per Pax & 5.84 & 5.84 & 5.84 & 5.84 & 5.84 & 14.01 & 7.62 & 9.75 & 10.61 & 14.01 & 7.01 & 7.01 & 7.01 & 7.01 & 7.01 & 23.21 & 4.80 & 9.29 & 13.77 & 18.26 \\
\hline \multicolumn{21}{|l|}{ Pax Charges } \\
\hline Passenger & 2899.48 & 1740.38 & 2029.29 & 2319.93 & 2899.48 & 1335.56 & 667.78 & 934.20 & 1069.14 & 1335.56 & 3525.74 & 1762.87 & 1762.87 & 1762.87 & 3525.74 & 2300.90 & 1127.96 & 1420.33 & 1691.94 & 1982.58 \\
\hline Transfer Pax & 0.00 & 0.00 & 0.00 & 0.00 & 0.00 & 0.00 & 0.00 & 0.00 & 0.00 & 0.00 & 0.00 & 0.00 & 0.00 & 0.00 & 0.00 & 0.00 & 0.00 & 0.00 & 0.00 & 0.00 \\
\hline Security & 378.87 & 378.87 & 378.87 & 378.87 & 378.87 & 1818.23 & 1818.23 & 1818.23 & 1818.23 & 1818.23 & 1105.47 & 1105.47 & 1105.47 & 1105.47 & 1105.47 & 51.90 & 50.17 & 50.17 & 50.17 & 50.17 \\
\hline Transfer Security & 0.00 & 0.00 & 0.00 & 0.00 & 0.0 & 0.6 & 0.00 & 0.00 & 0.0 & 0.00 & 0.00 & 0.00 & 0 & 0.6 & 0.00 & 0.00 & 0.0 & 0.00 & 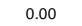 & 0.00 \\
\hline Infrastructure & 24.22 & 24.22 & 24.22 & 24.22 & 24.22 & 0.00 & 0.00 & 0.00 & 0.00 & 0.00 & 0.00 & 0.00 & 0.00 & 0.0 & . & 20.76 & 20.76 & 20.76 & 20.76 & 20.76 \\
\hline Gov. Taxes & 0.00 & 0.00 & 0.00 & 0.00 & 0.00 & 0.00 & 0.00 & 0.00 & 0.00 & 0.00 & 0.00 & 0.00 & 0.00 & 0.00 & 0.00 & 0.00 & 0.00 & 0.00 & 0.00 & 0.00 \\
\hline Total Pax Charge & $\begin{array}{l}3303.00 \\
1909\end{array}$ & $\begin{array}{l}2143.00 \\
1230\end{array}$ & 2432.00 & $\begin{array}{l}2723.00 \\
1574\end{array}$ & 3303.00 & $\begin{array}{l}3154.00 \\
18.22\end{array}$ & $\begin{array}{l}2486.00 \\
2437\end{array}$ & $\begin{array}{l}2752.00 \\
15.91\end{array}$ & 2887.00 & 3154.00 & 4631.00 & 2868.00 & 2868.00 & 2868.00 & 4631.00 & 2374.00 & 1199.00 & 1491.00 & 1763.00 & 2054.00 \\
\hline & & & & & & & & & & & 26.77 & & & & & 13.72 & 6.93 & 8.62 & 10.19 & 11.87 \\
\hline $\begin{array}{l}\text { Total harges } \\
\text { Average Per Pax }\end{array}$ & $\begin{array}{l}4313.00 \\
24.93\end{array}$ & $\begin{array}{l}3153.00 \\
18.23\end{array}$ & $\begin{array}{l}3442.00 \\
19.90\end{array}$ & $\begin{array}{l}3733.00 \\
21.58\end{array}$ & $\begin{array}{l}4313.00 \\
24.93\end{array}$ & $\begin{array}{c}5578.00 \\
32.24\end{array}$ & $\begin{array}{c}3805.00 \\
21.99\end{array}$ & $\begin{array}{l}4440.00 \\
25.66\end{array}$ & $\begin{array}{l}4722.00 \\
27.30\end{array}$ & $\begin{array}{c}5578.00 \\
32.24\end{array}$ & $\begin{array}{c}5843.00 \\
33.78\end{array}$ & $\begin{array}{c}4080.00 \\
23.59\end{array}$ & $\begin{array}{l}4080.00 \\
23.59\end{array}$ & $\begin{array}{c}4080.00 \\
23.59\end{array}$ & $\begin{array}{l}5843.00 \\
33.78\end{array}$ & $\begin{array}{l}6389.90 \\
36.93\end{array}$ & $\begin{array}{l}2030.00 \\
11.73\end{array}$ & $\begin{array}{l}3098.00 \\
17.91\end{array}$ & 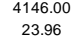 & $\begin{array}{l}5212.00 \\
30.13\end{array}$ \\
\hline
\end{tabular}

\begin{tabular}{|c|c|c|}
\hline issumptions & Currency. EUR 1.0 & \\
\hline $\begin{array}{l}\text { Airline: Lutthansa } \\
\text { Aircraft: A340-300 } \\
\text { Route: Intra EU }\end{array}$ & $\begin{array}{l}\text { MTOW: } 271 \text { tonnes } \\
\text { MLW: } 190 \text { tonnes }\end{array}$ & $\begin{array}{l}\text { Capacity: } 247 \\
\text { Load Factor: } \\
\text { Passengers: } 173\end{array}$ \\
\hline
\end{tabular}


Table 8: Calculations for Lufthansa A330-300 incentive scheme benchmarking exercise - standard operations

\begin{tabular}{|c|c|c|c|c|c|c|c|c|c|c|c|c|c|c|c|c|c|c|c|c|}
\hline \multirow{2}{*}{$\begin{array}{l}\text { Airport } \\
\text { Valid From } \\
\text { Runwway Charges }\end{array}$} & \multicolumn{5}{|c|}{ MLA } & \multicolumn{5}{|c|}{ NCE } & \multicolumn{5}{|c|}{ BRU } & \multicolumn{5}{|c|}{ BHX } \\
\hline & 0 & 1 & 2 & 3 & 4 & 0 & 1 & 2 & 3 & 4 & 0 & 1 & 2 & 3 & 4 & 0 & 1 & 2 & 3 & 4 \\
\hline & 866.36 & 866.36 & 866.36 & 866.36 & 866.36 & 1457.33 & 364.33 & 728.67 & 1457.33 & 1457.33 & 773.50 & 773.50 & 773.50 & 773.50 & 773.50 & 2722.87 & 0.00 & 667.15 & 1334.30 & 2001.44 \\
\hline Enuironment & 0.00 & 0.00 & 0.00 & 0.00 & 0.00 & -211.50 & -47.55 & -102.20 & -211.50 & -211.50 & -38.68 & -38.68 & -38.68 & -38.68 & -38.68 & 0.00 & 0.00 & 0.00 & 0.00 & 0.00 \\
\hline Terminal Nav. & 0.00 & 0.00 & 0.00 & 0.00 & 0.00 & 788.37 & 788.37 & 788.37 & 788.37 & 788.37 & 535.34 & 535.34 & 535.34 & 535.34 & 535.34 & 729.09 & 714.56 & 714.56 & 714.56 & 714.56 \\
\hline Terminal Charges & & & & & & & & & & & & & & & & & & & & \\
\hline Infrastructure & 0.00 & 0.00 & 0.00 & 0.00 & 0.00 & 0.00 & 0.00 & 0.00 & 0.00 & 0.00 & 5.66 & 5.66 & 5.66 & 5.66 & 5.66 & 0.00 & 0.00 & 0.00 & 0.00 & 0.00 \\
\hline $\begin{array}{l}\text { Airbridge } \\
\text { ifcratterking }\end{array}$ & 0.00 & 0.00 & 0.00 & 0.00 & 0.00 & $\begin{array}{l}39.74 \\
0.70\end{array}$ & 39.74 & 39.74 & 39.74 & 39.74 & 0.00 & 0.00 & 0.00 & 0.00 & 0.00 & 0.00 & 0.00 & 0.00 & 0.00 & 0.00 \\
\hline Aircratt Parking & 0.00 & 0.00 & 0.00 & 0.00 & 0.00 & $\begin{array}{l}0.00 \\
070\end{array}$ & 0.00 & $\begin{array}{l}0.00 \\
155\end{array}$ & $\begin{array}{c}0.00 \\
070\end{array}$ & $\begin{array}{l}0.00 \\
070\end{array}$ & 0.00 & 0.00 & 0.00 & $\begin{array}{l}0.00 \\
0700\end{array}$ & $\begin{array}{l}0.00 \\
0750\end{array}$ & 0.00 & 0.00 & 0.00 & 0.00 & 0.00 \\
\hline Airport Charges & 866.00 & 866.00 & 866.00 & 866.00 & 866.00 & 2074.00 & 1145.00 & 1455.00 & 2074.00 & 2074.00 & 1276.00 & 1276.00 & 1276.00 & 1276.00 & 1276.00 & 3452.00 & 715.00 & 1382.00 & 2049.00 & 2716.00 \\
\hline Average Per Pax & 5.59 & 5.59 & 5.59 & 5.59 & 5.59 & 13.38 & 7.39 & 9.38 & 13.38 & 13.38 & 8.23 & 8.23 & 8.23 & 8.23 & 8.23 & 22.27 & 4.61 & 8.91 & 13.22 & 17.52 \\
\hline $\begin{array}{l}\text { Pax Charges } \\
\text { Passenger }\end{array}$ & & & & & & & & & & & & & & & & & & & 151590 & \\
\hline $\begin{array}{l}\text { Tassenger } \\
\text { Transfer Pax }\end{array}$ & $\begin{array}{c}2598.80 \\
0.00\end{array}$ & $\begin{array}{c}1818.15 \\
0.00\end{array}$ & $\begin{array}{c}2018.55 \\
0.00\end{array}$ & $\begin{array}{c}233.7 .40 \\
0.00\end{array}$ & $\begin{array}{c}259.700 \\
0.00\end{array}$ & $\begin{array}{c}1196.60 \\
0.00\end{array}$ & $\begin{array}{c}50.30 \\
0.00\end{array}$ & $\begin{array}{c}83.00 \\
0.00\end{array}$ & $\begin{array}{c}1196.60 \\
0.00\end{array}$ & $\begin{array}{c}1196.60 \\
0.00\end{array}$ & $\begin{array}{c}3515.800 \\
0.00\end{array}$ & $\begin{array}{l}159 / 4.45 \\
0.00\end{array}$ & $\begin{array}{c}2366.95 \\
0.00\end{array}$ & $\begin{array}{c}2369.95 \\
0.00\end{array}$ & $\begin{array}{c}3158.00 \\
0.00\end{array}$ & $\begin{array}{c}2061.50 \\
0.00\end{array}$ & $\begin{array}{l}1010.60 \\
0.00\end{array}$ & $\begin{array}{c}12 / 2.255 \\
0.00\end{array}$ & $\begin{array}{c}1515.90 \\
0.00\end{array}$ & 0.00 \\
\hline Security & 339.45 & 339.45 & 339.45 & 339.45 & 339.45 & 1629.05 & 1629.05 & 1629.05 & 1629.05 & 1629.05 & 990.45 & 990.45 & 990.45 & 990.45 & 990.45 & 46.50 & 44.95 & 44.95 & 44.95 & 44.95 \\
\hline Transfer Security & 0.00 & 0.00 & 0.00 & 0.00 & 0.00 & 0.00 & 0.00 & 0.00 & 0.00 & 0.00 & 0.00 & 0.00 & 0.00 & 0.00 & 0.00 & 0.00 & 0.00 & 0.00 & 0.00 & 0.00 \\
\hline Infrastructure & 21.70 & 21.70 & 21.70 & 21.70 & 21.70 & 0.00 & 0.00 & 0.00 & 0.00 & 0.00 & 0.00 & 0.00 & 0.00 & 0.00 & 0.00 & 18.60 & 18.60 & 18.60 & 18.60 & 18.60 \\
\hline Gou. Taxes & 0.00 & 0.00 & 0.00 & 0.00 & 0.00 & 0.00 & 0.00 & 0.00 & 0.00 & 0.00 & 0.00 & 0.00 & 0.00 & 0.00 & 0.00 & 0.00 & 0.00 & 0.00 & 0.00 & 0.00 \\
\hline $\begin{array}{l}\text { Total Pax Charge } \\
\text { Average Pax Charge }\end{array}$ & $\begin{array}{c}2959.00 \\
19.09\end{array}$ & $\begin{array}{c}2179.00 \\
14.06\end{array}$ & $\begin{array}{c}2440.00 \\
15.74\end{array}$ & $\begin{array}{c}2699.00 \\
17.41\end{array}$ & $\begin{array}{c}2959.00 \\
19.09\end{array}$ & $\begin{array}{c}2826.00 \\
18.23\end{array}$ & $\begin{array}{c}2227.00 \\
14.37\end{array}$ & $\begin{array}{c}2466.00 \\
15.91\end{array}$ & $\begin{array}{c}2826.00 \\
18.23\end{array}$ & $\begin{array}{c}2826.00 \\
18.23\end{array}$ & $\begin{array}{c}4149.00 \\
26.77\end{array}$ & $\begin{array}{c}2570.00 \\
16.58\end{array}$ & $\begin{array}{c}3360.00 \\
21.68\end{array}$ & $\begin{array}{c}3360.00 \\
21.68\end{array}$ & $\begin{array}{c}4149.00 \\
26.77\end{array}$ & $\begin{array}{c}2127.00 \\
13.72\end{array}$ & $\begin{array}{c}1074.00 \\
6.93\end{array}$ & $\begin{array}{c}1336.00 \\
8.62\end{array}$ & $\begin{array}{c}1579.00 \\
10.19\end{array}$ & $\begin{array}{c}1840.00 \\
11.87\end{array}$ \\
\hline \begin{tabular}{|l|} 
Total Charges \\
Average Per Pax
\end{tabular} & $\begin{array}{c}3825.00 \\
24.68\end{array}$ & $\begin{array}{c}3046.00 \\
19.65\end{array}$ & $\begin{array}{l}3306.00 \\
21.33\end{array}$ & $\begin{array}{c}3565.00 \\
23.00\end{array}$ & $\begin{array}{c}3825.00 \\
24.68\end{array}$ & $\begin{array}{l}4900.00 \\
31.61\end{array}$ & $\begin{array}{c}3372.00 \\
21.76\end{array}$ & $\begin{array}{c}3921.00 \\
25.29\end{array}$ & $\begin{array}{c}4900.00 \\
31.61\end{array}$ & $\begin{array}{l}4900.00 \\
31.61\end{array}$ & $\begin{array}{l}5425.00 \\
35.00\end{array}$ & $\begin{array}{c}3846.00 \\
24.81\end{array}$ & $\begin{array}{c}4636.00 \\
29.91\end{array}$ & $\begin{array}{l}4636.00 \\
29.91\end{array}$ & $\begin{array}{c}5425.00 \\
35.00\end{array}$ & $\begin{array}{c}5579.00 \\
35.99\end{array}$ & $\begin{array}{c}1789.00 \\
11.54\end{array}$ & $\begin{array}{c}2718.00 \\
17.53\end{array}$ & $\begin{array}{c}3628.00 \\
23.41\end{array}$ & $\begin{array}{l}4556.00 \\
29.39\end{array}$ \\
\hline
\end{tabular}

\begin{tabular}{|c|c|c|}
\hline mptetic & Currency, EUR $1 \mathrm{E}$ & \\
\hline \begin{tabular}{|l} 
Airline: Luthansa \\
Aircraft: A330-300
\end{tabular} & $\begin{array}{l}\text { MTOW: } 233 \text { tonnes } \\
\text { MLW: } 180 \text { tonnes }\end{array}$ & $\begin{array}{l}\text { Capacity. } 221 \\
\text { Load Factor: } 70 \%\end{array}$ \\
\hline
\end{tabular}


Table 9: Calculations for Lufthansa A330-300 incentive scheme benchmarking exercise strategic operations

\begin{tabular}{|c|c|c|c|c|c|c|c|c|c|c|c|c|c|c|c|c|c|c|c|c|}
\hline $\begin{array}{c}\text { Airport } \\
\text { Year }\end{array}$ & 0 & 1 & $\frac{\text { MLA }}{2}$ & 3 & 4 & 0 & 1 & $\frac{\text { NCE }}{2}$ & 3 & 4 & 0 & 1 & $\frac{\text { BRU }}{2}$ & 3 & 4 & 0 & 1 & $\frac{\mathrm{BHX}}{2}$ & 3 & 4 \\
\hline \multicolumn{21}{|l|}{ Runway Charges } \\
\hline $\begin{array}{l}\text { Landing } \\
\text { Enviroment }\end{array}$ & 866.36 & $\begin{array}{l}866.36 \\
0.00\end{array}$ & 866.36 & 866.36 & 866.36 & 1457.33 & 364.33 & $\begin{array}{r}728.67 \\
.-1020\end{array}$ & 874.40 & $\begin{array}{l}1457.33 \\
0.50\end{array}$ & $\begin{array}{r}773.50 \\
3868\end{array}$ & $\begin{array}{r}773.50 \\
3068\end{array}$ & $\begin{array}{r}773.50 \\
3268\end{array}$ & $\begin{array}{r}773.50 \\
3260\end{array}$ & 2001.44 & 2722.87 & 0.00 & 667.15 & 1334.30 & 2001.44 \\
\hline 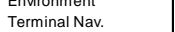 & 0.00 & 0.00 & 0.00 & 0.00 & 0.00 & $\begin{array}{l}-21.1 .00 \\
788.37\end{array}$ & $\begin{array}{l}-478.53 \\
788.37\end{array}$ & $\begin{array}{l}-102.20 \\
788.37\end{array}$ & $\begin{array}{l}-144.00 \\
788.37\end{array}$ & $\begin{array}{l}-211.50 \\
788.37\end{array}$ & $\begin{array}{l}-38.08 \\
535.34\end{array}$ & $\begin{array}{l}-30.08 \\
535.34\end{array}$ & 535.34 & $\begin{array}{l}-38.68 \\
535.34\end{array}$ & 7.006 & 7.000 & $\begin{array}{l}0.00 \\
71456\end{array}$ & $\begin{array}{l}0.00 \\
71456\end{array}$ & $\begin{array}{l}0.00 \\
77456\end{array}$ & $\begin{array}{l}0.00 \\
7145\end{array}$ \\
\hline \multicolumn{21}{|l|}{ Terminal Charges } \\
\hline Infrastructure & 0.00 & 0.00 & 0.00 & 0.00 & 0.00 & 0.00 & 0.00 & 0.00 & 0.00 & 0.00 & 5.66 & 5.66 & 5.66 & 5.66 & 0.00 & 0.00 & 0.00 & 0.00 & 0.00 & 0.00 \\
\hline Airbridge & 0.00 & 0.00 & 0.00 & 0.00 & 0.00 & 39.74 & 39.74 & 39.74 & 39.74 & 39.74 & 0.00 & 0.00 & 0.00 & 0.00 & 0.00 & 0.00 & 0.00 & 0.00 & 0.00 & 0.00 \\
\hline Aircraft Parking & 0.00 & 0.00 & 0.00 & 0.00 & 0.00 & 0.00 & 0.00 & 0.00 & 0.00 & 0.00 & 0.00 & 0.00 & 0.00 & 0.00 & 0.00 & 0.00 & 0.00 & 0.00 & 0.00 & 0.00 \\
\hline Airport Charges & 866.00 & 866.00 & 866.00 & 866.00 & 866.00 & 2074.00 & 1145.00 & 1455.00 & 1578.00 & 2074.00 & 1276.00 & 1276.00 & 1276.00 & 1276.00 & 2716.00 & 3452.00 & 715.00 & 1382.00 & 2049.00 & 2716.00 \\
\hline Average Per Pax & 5.59 & 5.59 & 5.59 & 5.59 & 5.59 & 13.38 & 7.39 & 9.38 & 10.18 & 13.38 & 8.23 & 8.23 & 8.23 & 8.23 & 17.52 & 22.27 & 4.61 & 8.91 & 13.22 & 17.52 \\
\hline \multicolumn{21}{|l|}{$\begin{array}{l}\text { Pax Charges } \\
\text { Passenoer }\end{array}$} \\
\hline Passenger & 2597.80 & 1559.30 & 1818.15 & 2078.55 & 2597.80 & 1196.60 & 598.30 & 837.00 & 957.90 & 1196.60 & 3158.90 & 1579.45 & 1579.45 & 1579.45 & 1776.30 & 2061.50 & 1010.60 & 1272.55 & 1515.90 & 1776.30 \\
\hline Transfer Pax & 0.00 & 0.00 & 0.00 & 0.00 & 0.00 & 0.00 & 0.00 & 0.00 & 0.00 & 0.00 & 0.00 & 0.00 & 0.00 & 0.00 & 0.00 & 0.00 & 0.00 & 0.00 & 0.00 & 0.00 \\
\hline Security & 339.45 & 339.45 & 339.45 & 339.45 & 339.45 & 1629.05 & 1629.05 & 1629.05 & 1629.05 & 1629.05 & 990.45 & 990.45 & 990.45 & 990.45 & 44.95 & 46.50 & 44.95 & 44.95 & 44.95 & 44.95 \\
\hline $\begin{array}{l}\text { Transfer Security } \\
\text { Infrastructure }\end{array}$ & $\begin{array}{l}0.00 \\
0.17\end{array}$ & $\begin{array}{l}0.00 \\
2170\end{array}$ & $\begin{array}{l}0.00 \\
2170\end{array}$ & $\begin{array}{l}0.00 \\
2170\end{array}$ & $\begin{array}{l}0.00 \\
2170\end{array}$ & 0.00 & 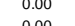 & 0.00 & $\begin{array}{l}0.00 \\
0.00\end{array}$ & 0 & 0.00 & o. 00 & 0.000 & 0.000 & 0.00 & $\begin{array}{l}0.00 \\
0.00\end{array}$ & $\begin{array}{l}0.00 \\
0.060\end{array}$ & 0.000 & 0.000 & 0 \\
\hline Govt. Taxes & $\begin{array}{l}2.10 \\
0.00\end{array}$ & 0.00 & $\begin{array}{l}0.10 \\
0.00\end{array}$ & $\begin{array}{l}0.00 \\
0.00\end{array}$ & $\begin{array}{l}2.10 \\
0.00\end{array}$ & 0.00 & 0.00 & 0.00 & 0.00 & 0.00 & 0.00 & 0.00 & 0.00 & 0.00 & $\begin{array}{l}18.00 \\
0.00\end{array}$ & $\begin{array}{l}18.00 \\
0.00\end{array}$ & 0.00 & 0.00 & 0.00 & $\begin{array}{l}18.00 \\
0.00\end{array}$ \\
\hline Total Pax Charge & 2959.00 & 1920.00 & 2179.00 & 2440.00 & 2959.00 & 2826.00 & 2227.00 & 2466.00 & 2587.00 & 2826.00 & 4149.00 & 2570.00 & 2570.00 & 2570.00 & 1840.00 & 2127.00 & 1074.00 & 1336.00 & 1579.00 & 1840.00 \\
\hline Average Pax Charge & 19.09 & 12.39 & 14.06 & 15.74 & 19.09 & 18.23 & 14.37 & 15.91 & 16.69 & 18.23 & 26.77 & 16.58 & 16.58 & 16.58 & 11.87 & 13.72 & 6.93 & 8.62 & 10.19 & 11.87 \\
\hline Total Charges & 3825.00 & 2787.00 & 3046.00 & 3306.00 & 3825.00 & 4900.00 & 3372.00 & 3921.00 & 4165.00 & 4900.00 & 5425.00 & 3846.00 & 3846.00 & 3846.00 & 4556.00 & 5579.00 & 1789.00 & 2718.00 & 3628.00 & 4556.00 \\
\hline Average Per Pax & 24.68 & 17.98 & 19.65 & 21.33 & 24.68 & 31.61 & 21.76 & 25.29 & 26.87 & 31.61 & 35.00 & 24.81 & 24.81 & 24.81 & 29.39 & 35.99 & 11.54 & 17.53 & 23.41 & 29.39 \\
\hline
\end{tabular}

\begin{tabular}{|c|c|c|}
\hline Assumptions & Currency: EUR $1 \mathrm{E}$ & \\
\hline $\begin{array}{l}\text { Airline: Lutthansa } \\
\text { Aircratt: } A 330-300 \\
\text { Route: }\end{array}$ & $\begin{array}{l}\text { MTOW: } 233 \text { tonnes } \\
\text { MLW: } 180 \text { tonnes }\end{array}$ & $\begin{array}{l}\text { Capacity: } 221 \\
\text { Load Factor: } 70 \% \\
\text { Passengers }\end{array}$ \\
\hline
\end{tabular}


Table 10: Calculations for Ryanair B737-800 incentive scheme benchmarking exercise - standard operations

\begin{tabular}{|c|c|c|c|c|c|c|c|c|c|c|c|c|c|c|c|c|c|c|c|c|}
\hline $\begin{array}{l}\text { Airport } \\
\text { Year }\end{array}$ & 0 & 1 & MLA & 3 & & & 1 & $\frac{\mathrm{NCE}}{2}$ & 2 & & & & BRU & & & & & $\frac{B H X}{2}$ & & \\
\hline & 年 & + & & 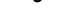 & 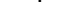 & 5 & + & & 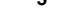 & ${ }^{4}$ & 0 & + & & 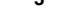 & 4 & 0 & 1 & 2 & ${ }^{\circ}$ & 4 \\
\hline \multicolumn{21}{|l|}{$\begin{array}{l}\text { Runway Charges } \\
\text { Landing }\end{array}$} \\
\hline Environment & $\begin{array}{c}20.000 \\
0.00\end{array}$ & 200.00 & 200.00 & $\begin{array}{c}238.88 \\
0.00\end{array}$ & $\begin{array}{l}238.88 \\
0.00\end{array}$ & $\begin{array}{l}268.93 \\
-34.86\end{array}$ & $\begin{array}{l}67.23 \\
-4.61\end{array}$ & $\begin{array}{l}133.47 \\
-14.69\end{array}$ & $\begin{array}{l}161.36 \\
-18.73\end{array}$ & $\begin{array}{l}266.93 \\
-34.86\end{array}$ & $\begin{array}{l}269.14 \\
-14.81\end{array}$ & $\begin{array}{l}296.14 \\
-14.81\end{array}$ & $\begin{array}{l}246.14 \\
-14.81\end{array}$ & $\begin{array}{l}266.14 \\
-14.81\end{array}$ & $\begin{array}{l}269.14 \\
-14.81\end{array}$ & $\begin{array}{l}782.97 \\
0.00\end{array}$ & $\begin{array}{l}0.00 \\
0.00\end{array}$ & $\begin{array}{l}191.84 \\
0.00\end{array}$ & $\begin{array}{l}383.69 \\
0.00\end{array}$ & $\begin{array}{l}575.52 \\
0.00\end{array}$ \\
\hline Terminal Nav. & 0.00 & 0.00 & 0.00 & 0.00 & 0.00 & 256.79 & 256.79 & 256.79 & 256.79 & 256.79 & 174.37 & 174.37 & 174.37 & 174.37 & 174.37 & 209.65 & 205.47 & 205.47 & 205.47 & 205.47 \\
\hline \multicolumn{21}{|l|}{ Terminal Charges } \\
\hline Infrastructure & 0.00 & 0.00 & 0.00 & 0.00 & 0.00 & 0.00 & 0.00 & 0.00 & 0.00 & 0.00 & 5.66 & 5.66 & 5.66 & 5.66 & 5.66 & 0.00 & 0.00 & 0.00 & 0.00 & 0.00 \\
\hline $\begin{array}{l}\text { Airrigdge } \\
\text { Aicrattrarking }\end{array}$ & $\begin{array}{l}0.00 \\
0.00\end{array}$ & $\begin{array}{l}0.00 \\
0.00\end{array}$ & $\begin{array}{l}0.00 \\
0.00\end{array}$ & $\begin{array}{l}0.00 \\
0.00\end{array}$ & $\begin{array}{l}0.00 \\
0.00\end{array}$ & $\begin{array}{l}0.00 \\
0.00\end{array}$ & $\begin{array}{l}0.00 \\
0.00\end{array}$ & $\begin{array}{l}0.00 \\
0.00\end{array}$ & $\begin{array}{l}0.00 \\
0.00\end{array}$ & $\begin{array}{l}0.00 \\
0.00\end{array}$ & $\begin{array}{l}0.00 \\
0.00\end{array}$ & $\begin{array}{l}0.00 \\
0.00\end{array}$ & $\begin{array}{l}0.00 \\
0.00\end{array}$ & $\begin{array}{l}0.00 \\
0.00\end{array}$ & $\begin{array}{l}0.00 \\
0.00\end{array}$ & $\begin{array}{l}0.00 \\
0.00\end{array}$ & $\begin{array}{l}0.00 \\
0.00\end{array}$ & $\begin{array}{l}0.00 \\
0.00\end{array}$ & $\begin{array}{l}0.00 \\
0.00\end{array}$ & $\begin{array}{l}0.00 \\
0.00\end{array}$ \\
\hline Airport Charges & 239.00 & 239.00 & 239.00 & 239.00 & 239.00 & 491.00 & 319.00 & 377.00 & 399.00 & 491.00 & 461.00 & 461.00 & 461.00 & 461.00 & 461.00 & 993.00 & 205.00 & 397.00 & 589.00 & 781.00 \\
\hline Average Per Pax & 1.81 & 1.81 & 1.81 & 1.81 & 1.81 & 3.72 & 2.42 & 2.85 & 3.03 & 3.72 & 3.50 & 3.50 & 3.50 & 3.50 & 3.50 & 7.52 & 1.56 & 3.01 & 4.46 & 5.92 \\
\hline \multicolumn{21}{|l|}{$\begin{array}{l}\text { Pax Charges } \\
\text { Passenger }\end{array}$} \\
\hline $\begin{array}{l}\text { Passenger } \\
\text { Transfer Pax }\end{array}$ & $\begin{array}{l}2212.32 \\
0.00\end{array}$ & 1327.92 & 1548.36 & 1770.12 & 2212.32 & 1019.04 & 509.52 & 712.80 & 815.76 & 1019.04 & 2690.16 & 1345.08 & 1345.08 & 1345.08 & 2690.16 & 1755.60 & 528.00 & 665.28 & 792.00 & 929.28 \\
\hline $\begin{array}{l}\text { Transfer Pax } \\
\text { Security }\end{array}$ & $\begin{array}{l}0.00 \\
289.08\end{array}$ & $\begin{array}{l}0.00 \\
289.08\end{array}$ & $\begin{array}{l}0.00 \\
289.08\end{array}$ & $\begin{array}{l}0.00 \\
289.08\end{array}$ & $\begin{array}{l}0.00 \\
289.08\end{array}$ & $\begin{array}{l}0.00 \\
1387.32\end{array}$ & $\begin{array}{l}0.00 \\
1387.32\end{array}$ & $\begin{array}{l}0.00 \\
1387.32\end{array}$ & $\begin{array}{c}0.00 \\
1387.32\end{array}$ & $\begin{array}{l}0.00 \\
1387.32\end{array}$ & $\begin{array}{l}0.00 \\
843.48\end{array}$ & $\begin{array}{l}0.00 \\
843.48\end{array}$ & $\begin{array}{l}0.00 \\
843.48\end{array}$ & $\begin{array}{l}0.00 \\
843.48\end{array}$ & $\begin{array}{l}0.00 \\
843.48\end{array}$ & $\begin{array}{l}0.00 \\
39.60\end{array}$ & $\begin{array}{l}0.00 \\
38.28 \\
-\end{array}$ & $\begin{array}{l}0.00 \\
3828 \\
3828\end{array}$ & $\begin{array}{l}0.00 \\
3828\end{array}$ & $\begin{array}{l}0.00 \\
3828 \\
3828\end{array}$ \\
\hline Transfer Security & 0.00 & 0.00 & 0.00 & 0.00 & $\begin{array}{l}203000 \\
0.00\end{array}$ & 0.00 & 0.00 & 0.00 & 0.00 & 0.00 & 0.00 & 0.00 & 0.00 & 0.00 & 0.00 & 0.00 & 0.00 & $0.0 \mathrm{Cl}$ & 0.00 & 0.00 \\
\hline $\begin{array}{l}\text { Infrastructure } \\
\text { Pate }\end{array}$ & 18.48 & 18.48 & 18.48 & 18.48 & 18.48 & 0.00 & 0.00 & 0.00 & 0.00 & 0.00 & 0.00 & 0.00 & 0.00 & 0.00 & 18.60 & 15.84 & 15.84 & 15.84 & 15.84 & 15.84 \\
\hline Govt. Taxes & 0.00 & 0.00 & 0.00 & 0.00 & 0.00 & 0.00 & 0.00 & 0.00 & 0.00 & 0.00 & 0.00 & 0.00 & 0.00 & 0.00 & 0.00 & 0.00 & 0.00 & 0.00 & 0.00 & 0.00 \\
\hline Total Pax Charge & 2520.00 & 1635.00 & 1856.00 & 2078.00 & 2520.00 & 2406.00 & 1897.00 & 2100.00 & 2203.00 & 2406.00 & 3534.00 & 2189.00 & 2189.00 & 2189.00 & 3534.00 & 1811.00 & 582.00 & 719.00 & 846.00 & 983.00 \\
\hline Average Pax Charge & 19.09 & 12.39 & 14.06 & ${ }^{15.74}$ & 19.09 & 18.23 & 14.37 & 15.91 & 16.69 & 18.23 & 26.77 & 16.58 & 16.58 & 16.58 & 26.77 & 13.72 & 4.41 & 5.45 & 6.41 & 7.45 \\
\hline $\begin{array}{l}\text { Total Charges } \\
\text { Average Per Pax }\end{array}$ & $\begin{array}{l}2759.00 \\
20.90\end{array}$ & $\begin{array}{l}1874.00 \\
14.20\end{array}$ & $\begin{array}{l}2095.00 \\
15.87\end{array}$ & $\begin{array}{l}2317.00 \\
175\end{array}$ & $\begin{array}{l}2759.00 \\
20.90\end{array}$ & $\begin{array}{l}2897.00 \\
21.95\end{array}$ & $\begin{array}{l}2216.00 \\
16.79\end{array}$ & $\begin{array}{l}2477.00 \\
1876\end{array}$ & $\begin{array}{r}2603.00 \\
10.72\end{array}$ & $\begin{array}{l}2897.00 \\
21.95\end{array}$ & $\begin{array}{l}3995.00 \\
3027\end{array}$ & 2650.00 & 2650.00 & 2650.00 & $\begin{array}{l}3995.00 \\
30.27\end{array}$ & $\begin{array}{l}2804.00 \\
21.24\end{array}$ & $\begin{array}{c}788.00 \\
5.07\end{array}$ & 1117.00 & 1435.00 & 1764.00 \\
\hline
\end{tabular}

\begin{tabular}{|c|c|c|}
\hline 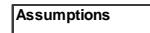 & $\begin{array}{l}\text { Currency: } \\
\end{array}$ & \\
\hline $\begin{array}{l}\text { Airline: Ryanair } \\
\text { Arcraft: B3733-800 } \\
\text { Route: Intra EU }\end{array}$ & $\begin{array}{l}\text { MTOW: } 66.99 \text { tones } \\
\text { MLW: } 66.3 \text { tonnes }\end{array}$ & $\begin{array}{l}\text { Capacity: } 189 \\
\text { Load Factor: } 70 \% \\
\text { Passengers: } 132\end{array}$ \\
\hline
\end{tabular}


Table 11: Calculations for Ryanair B737-800 incentive scheme benchmarking exercise strategic operations

\begin{tabular}{|c|c|c|c|c|c|c|c|c|c|c|c|c|c|c|c|c|c|c|c|c|}
\hline \multirow{2}{*}{$\begin{array}{l}\text { Airportr } \\
\text { Year }\end{array}$} & \multicolumn{5}{|c|}{ MLA } & \multicolumn{5}{|c|}{ NCE } & \multicolumn{5}{|c|}{ BRU } & \multicolumn{5}{|c|}{ BHX } \\
\hline & 0 & 1 & 2 & 3 & 4 & 0 & 1 & 2 & 3 & 4 & 0 & 1 & 2 & 3 & 4 & 0 & 1 & 2 & 3 & 4 \\
\hline Runway Charges & & & & & & & & & & & & & & & & & & & & \\
\hline $\begin{array}{l}\text { Landing } \\
\text { Enviroment }\end{array}$ & 238.88 & 238.88 & 238.88 & 238.88 & 238.88 & 268.93 & 67.23 & 134.47 & 161.36 & 268.93 & 296.14 & 296.14 & 296.14 & 296.14 & 296.14 & 782.97 & 0.00 & 191.84 & 383.69 & 575.52 \\
\hline $\begin{array}{l}\text { Enironment } \\
\text { Terminal Nav. }\end{array}$ & $\begin{array}{l}0.00 \\
0.00\end{array}$ & 0.00 & 0.00 & 0.00 & 0.00 & $\begin{array}{l}-34.86 \\
25679\end{array}$ & $\begin{array}{l}-4.61 \\
-25679\end{array}$ & $\begin{array}{l}-14.69 \\
25679\end{array}$ & $\begin{array}{l}-18.73 \\
25679\end{array}$ & $\begin{array}{r}-34.86 \\
2567\end{array}$ & $\begin{array}{l}-14.81 \\
1.7437\end{array}$ & $\begin{array}{l}-14.81 \\
-1.87\end{array}$ & $\begin{array}{l}-14.81 \\
-1.87\end{array}$ & $\begin{array}{l}-14.81 \\
-1.87\end{array}$ & -14.81 & 0.00 & 0.00 & 0.00 & 0.00 & 0.00 \\
\hline $\begin{array}{l}\text { Terminal Nav. } \\
\text { Terminal Charges }\end{array}$ & & & & & & & & & & & 174.37 & 174.37 & & & 174.37 & & 205.47 & & & 205.47 \\
\hline Infrastructure & 0.00 & 0.00 & 0.00 & 0.00 & 0.00 & 0.00 & 0.00 & 0.00 & 0.00 & 0.00 & 5.66 & 5.66 & 5.66 & 5.66 & 5.66 & 0.00 & 0.00 & 0.00 & 000 & 000 \\
\hline Airbridge & 0.00 & 0.00 & 0.00 & 0.00 & 0.00 & 0.00 & 0.00 & 0.00 & 0.00 & 0.00 & 0.00 & 0.00 & 0.00 & 0.00 & 0.00 & 0.00 & 0.00 & 0.00 & 0.00 & 0.00 \\
\hline Aircraft Parking & 0.00 & 0.00 & 0.00 & 0.00 & 0.00 & 0.00 & 0.00 & 0.00 & 0.00 & 0.00 & 0.00 & 0.00 & 0.00 & 0.00 & 0.00 & 0.00 & 0.00 & 0.00 & 0.00 & 0.00 \\
\hline Airport Charges & 239.00 & 239.00 & 239.00 & 239.00 & 239.00 & 491.00 & 319.00 & 377.00 & 399.00 & 491.00 & 461.00 & 461.00 & 461.00 & 461.00 & 461.00 & 993.00 & 205.00 & 397.00 & 589.00 & 781.00 \\
\hline Average Per Pax & 1.81 & 1.81 & 1.81 & 1.81 & 1.81 & 3.72 & 2.42 & 2.85 & 3.03 & 3.72 & 3.50 & 3.50 & 3.50 & 3.50 & 3.50 & 7.52 & 1.56 & 3.01 & 4.46 & 5.92 \\
\hline Pax Charges & & & & & & & & & & & & & & & & & & & & \\
\hline $\begin{array}{l}\text { Passenger } \\
\end{array}$ & 2212.32 & 1327.92 & 1548.36 & 1770.12 & 2212.32 & 1019.04 & 509.52 & 712.80 & 815.76 & 1019.04 & 2690.16 & 1345.08 & 1345.08 & 1345.08 & 2690.16 & 1755.60 & 528.00 & 665.28 & 792.00 & 929.28 \\
\hline $\begin{array}{l}\text { Transfer Pax } \\
\text { Security }\end{array}$ & 289.08 & $\begin{array}{l}0.00 \\
289.08\end{array}$ & $\begin{array}{l}0.00 \\
289.08\end{array}$ & $\begin{array}{l}0.00 \\
289.08\end{array}$ & $\begin{array}{l}0.00 \\
289.08\end{array}$ & $\begin{array}{l}0.00 \\
1387.32\end{array}$ & $\begin{array}{l}0.00 \\
1387.32\end{array}$ & $\begin{array}{l}0.00 \\
1387.32\end{array}$ & $\begin{array}{l}0.00 \\
1387.32\end{array}$ & $\begin{array}{l}0.00 \\
1387.32\end{array}$ & $\begin{array}{l}0.00 \\
843.48\end{array}$ & $\begin{array}{l}0.000 \\
843.48\end{array}$ & $\begin{array}{l}0.00 \\
843.48\end{array}$ & $\begin{array}{l}0.00 \\
843.48\end{array}$ & $\begin{array}{l}0.00 \\
84348\end{array}$ & $\begin{array}{l}0.00 \\
3960\end{array}$ & $\begin{array}{l}0.00 \\
3828\end{array}$ & $\begin{array}{l}0.00 \\
3828\end{array}$ & $\begin{array}{l}0.00 \\
3828\end{array}$ & $\begin{array}{l}0.00 \\
3828\end{array}$ \\
\hline Transfer Security & 0.00 & 0.00 & 0.00 & 0.00 & 0.00 & 0.00 & 0.00 & 0.00 & 0.00 & 0.00 & 0.00 & 0.00 & 000 & 0.0 & $0.00^{\circ}$ & 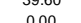 & 38.28 & 管 & 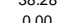 & 38.28 \\
\hline $\begin{array}{l}\text { Infrastructure } \\
\text { Intivecur }\end{array}$ & 18.48 & 18.48 & 18.48 & 18.48 & 18.48 & 0.00 & 0.00 & 0.00 & 0.00 & 0.00 & 0.00 & 0.00 & 0.00 & 0.00 & 1860 & 1584 & 1584 & 1584 & 1584 & 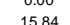 \\
\hline Govt. Taxes & 0.00 & 0.00 & 0.00 & 0.00 & 0.00 & 0.00 & 0.00 & 0.00 & 0.00 & 0.00 & 0.00 & 0.00 & 0.00 & 0.00 & 0.00 & 0.00 & 0.00 & 0.00 & 0.00 & $\begin{array}{l}1.084 \\
0.00\end{array}$ \\
\hline Total Pax Charge & 2520.00 & 1635.00 & 1856.00 & 2078.00 & 2520.00 & 2406.00 & 1897.00 & 2100.00 & 2203.00 & 2406.00 & 3534.00 & 2189.00 & 2189.00 & 2189.00 & 3534.00 & 1811.00 & 582.00 & 719.00 & 846.00 & 983.00 \\
\hline Average Pax Charge & 19.09 & 12.39 & 14.06 & 15.74 & 19.09 & 18.23 & 14.37 & 15.91 & 16.69 & 18.23 & 26.77 & 16.58 & 16.58 & 16.58 & 26.77 & 13.72 & 4.41 & 5.45 & 6.41 & 7.45 \\
\hline Total Charges & 2759.00 & 1874.00 & 2095.00 & 2317.00 & 2759.00 & 2897.00 & 2216.00 & 2477.00 & 2603.00 & 2897.00 & 3995.00 & 2650.00 & 2650.00 & 2650.00 & 3995.00 & 2804.00 & 788.00 & 1117.00 & 1435.00 & 1764.00 \\
\hline Average Per Pax & 20.90 & 14.20 & 15.87 & 17.55 & 20.90 & 21.95 & 16.79 & 18.76 & 19.72 & 21.95 & 30.27 & 20.08 & 20.08 & 20.08 & 30.27 & 21.24 & 5.97 & 8.46 & 10.87 & 13.37 \\
\hline
\end{tabular}

\begin{tabular}{|c|c|c|}
\hline Assumptions & Currency: EUR $1.00 \mathrm{E}$ & \\
\hline $\begin{array}{l}\text { Airline: Ryanair } \\
\text { Aircraft: } 3373-800 \\
\text { Route: Intra EU }\end{array}$ & $\begin{array}{l}\text { MroW: } 66.99 \text { tonnes } \\
\text { MLW: } 66.3 \text { tonnes }\end{array}$ & $\begin{array}{l}\text { Capacity: } 189 \\
\text { Load Factor: } 70 \% \\
\text { Passengers: } 132\end{array}$ \\
\hline
\end{tabular}




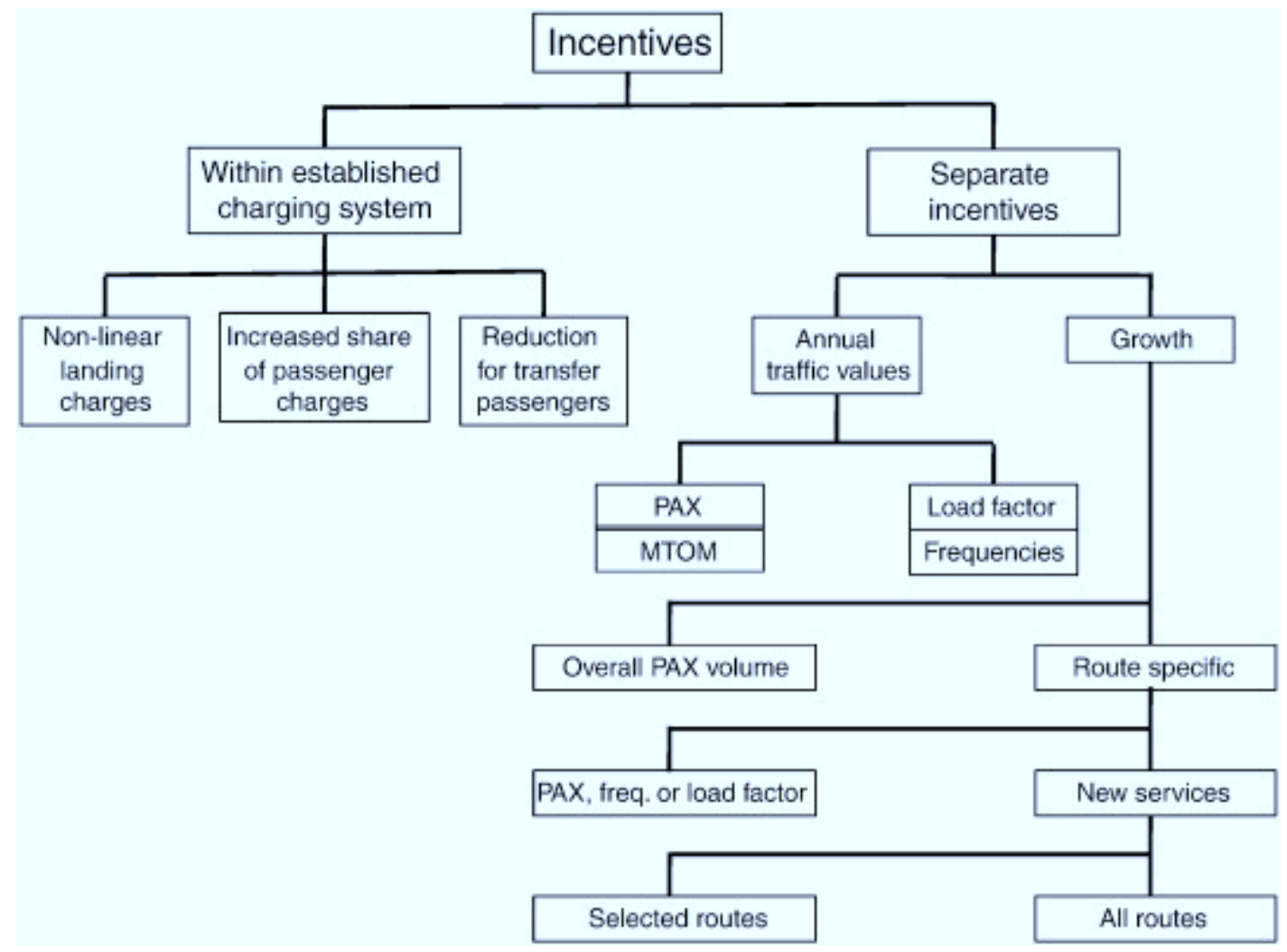

Figure 1. Overview of Incentive Schemes within airport charging systems

Source: Fichert and Klophaus (2011). 


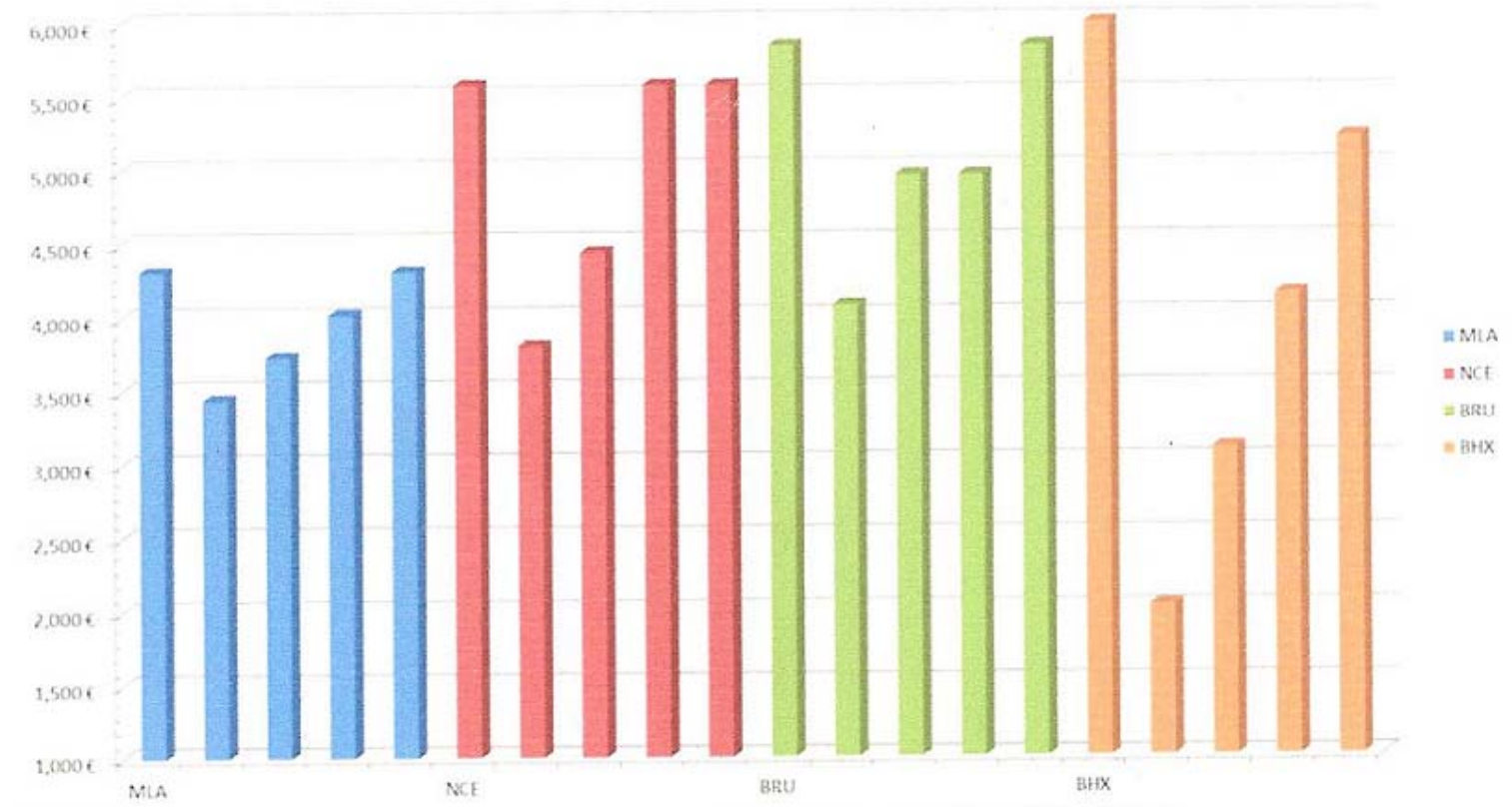

Figure 2: Total Charges for a LH A340- 300 operating with a "generic" incentive scheme, over a 4 year period, including a standard benchmark

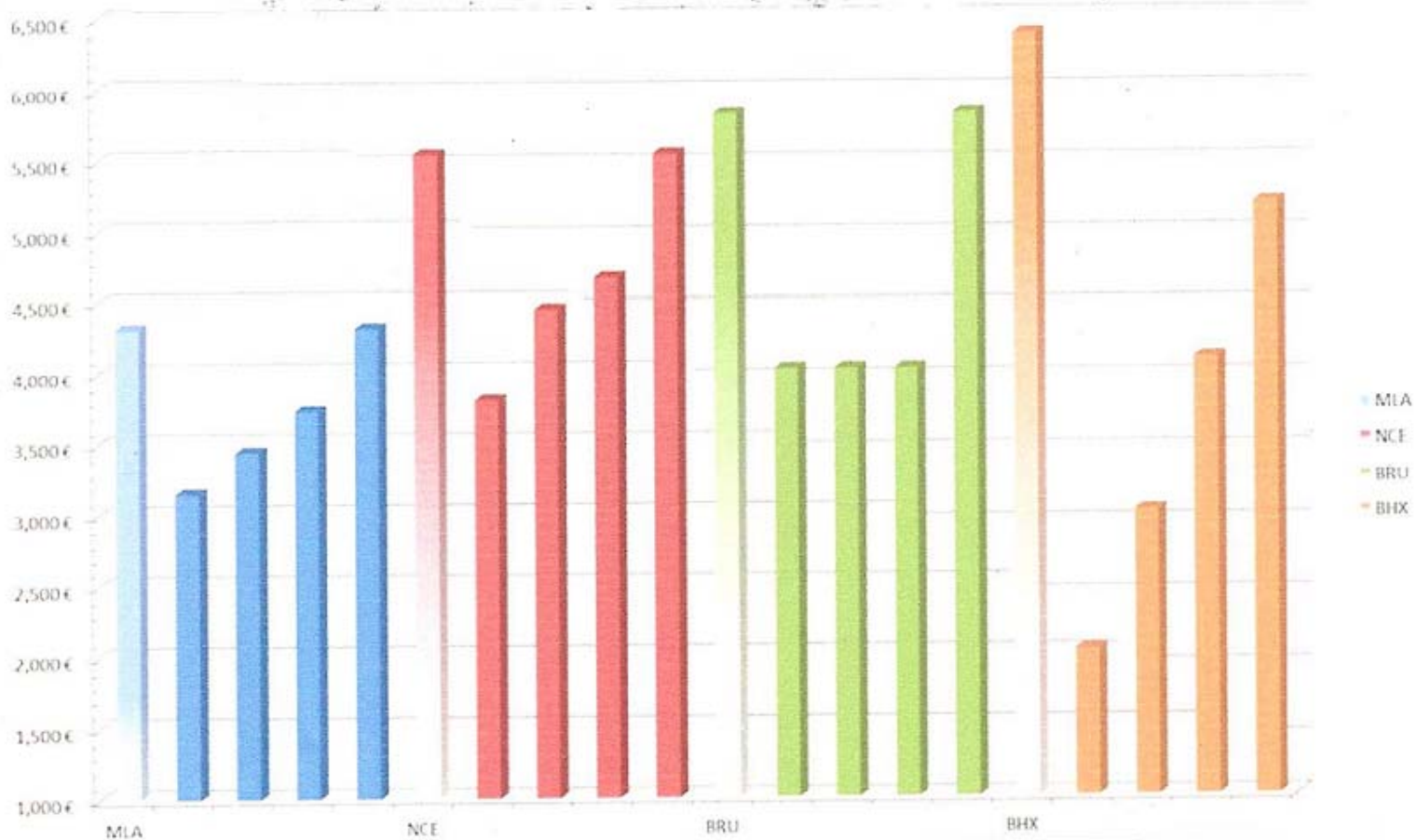

Figure 3: Total Charges for a LH A340- 300 operating with a "strategic" incentive scheme, over a 4 year period, including a standard benchmark 


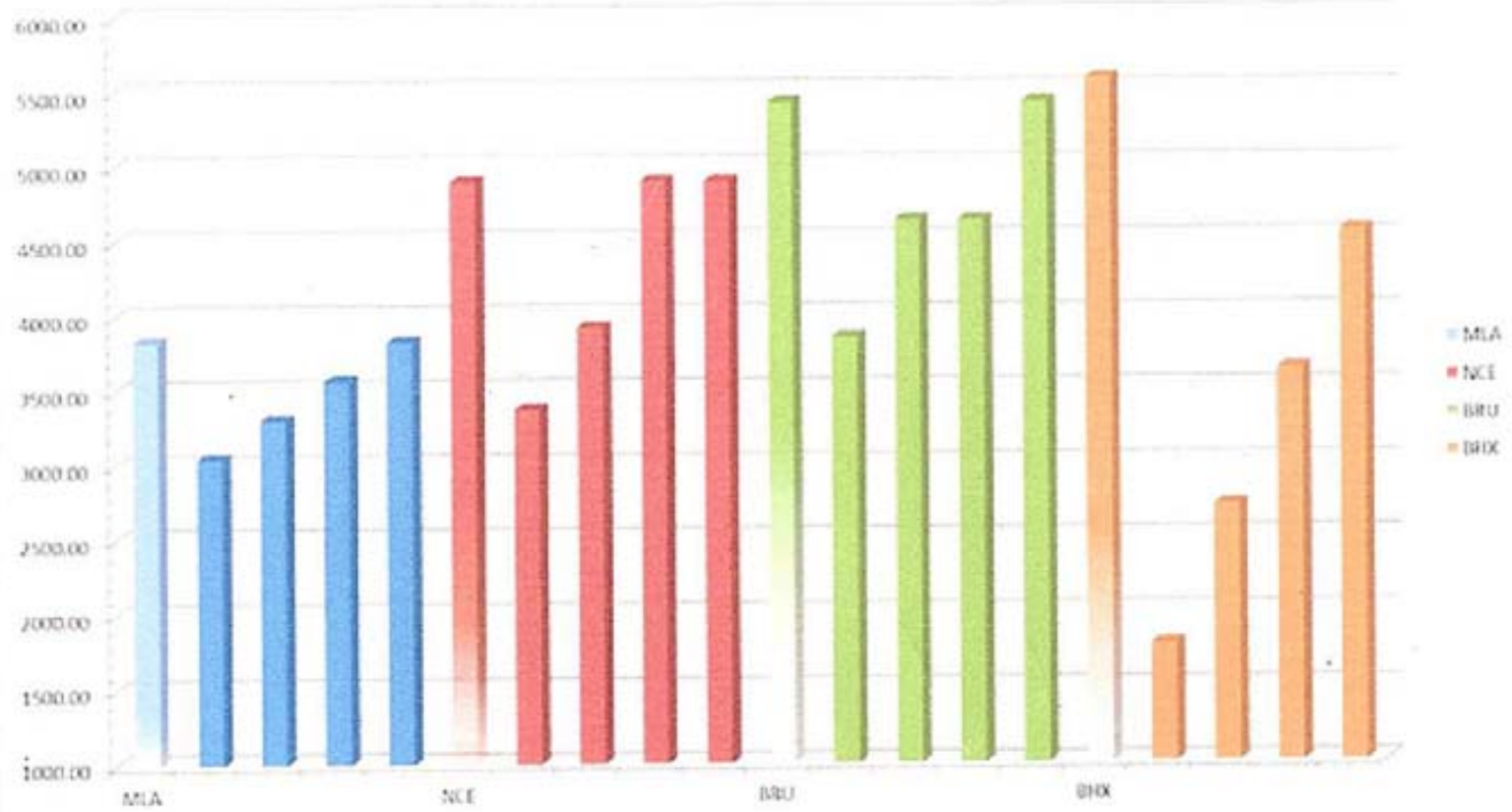

Figure 4: Total Charges for a LH A330- 300 operating with a "generic" incentive scheme, over a 4 year period, including a standard benchmark

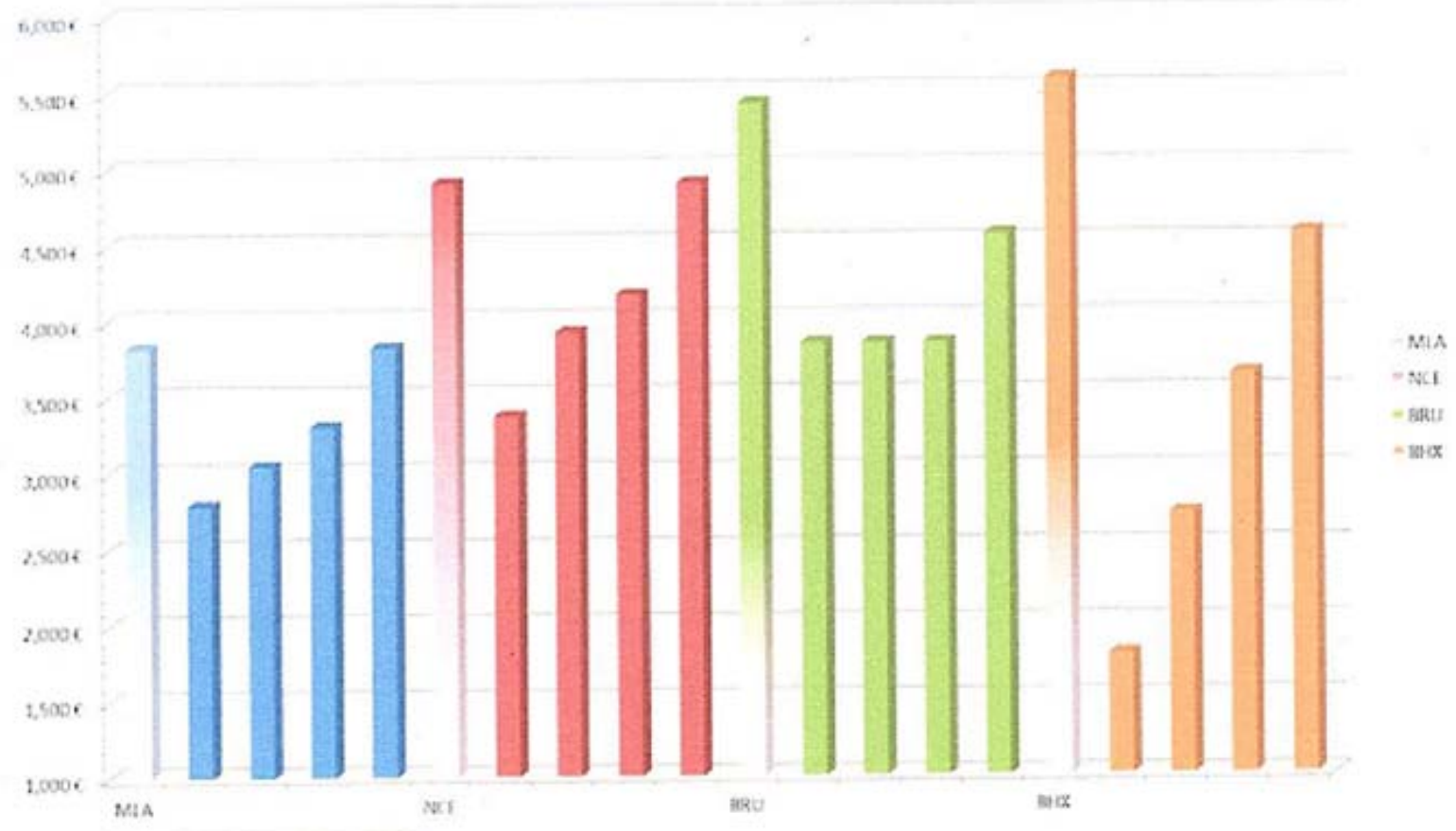

Figure 5: Total Charges for a LH A330- 300 operating with a "strategic" incentive scheme, over a 4 year period, including a standard benchmark 


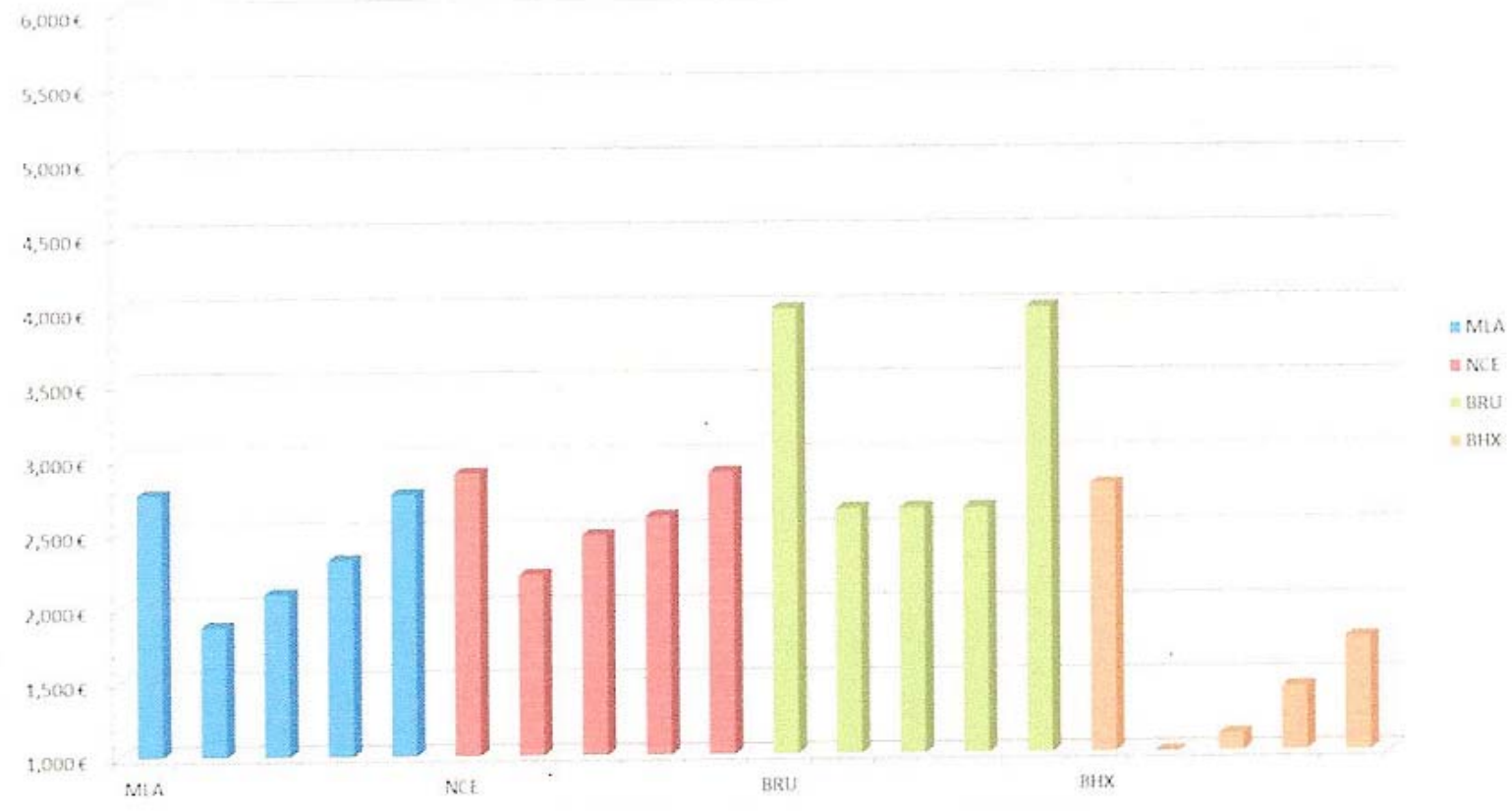

Figure 6: Total Charges for a FR B737-800 operating with a "generic" incentive scheme, over a 4 year period, including a standard benchmark

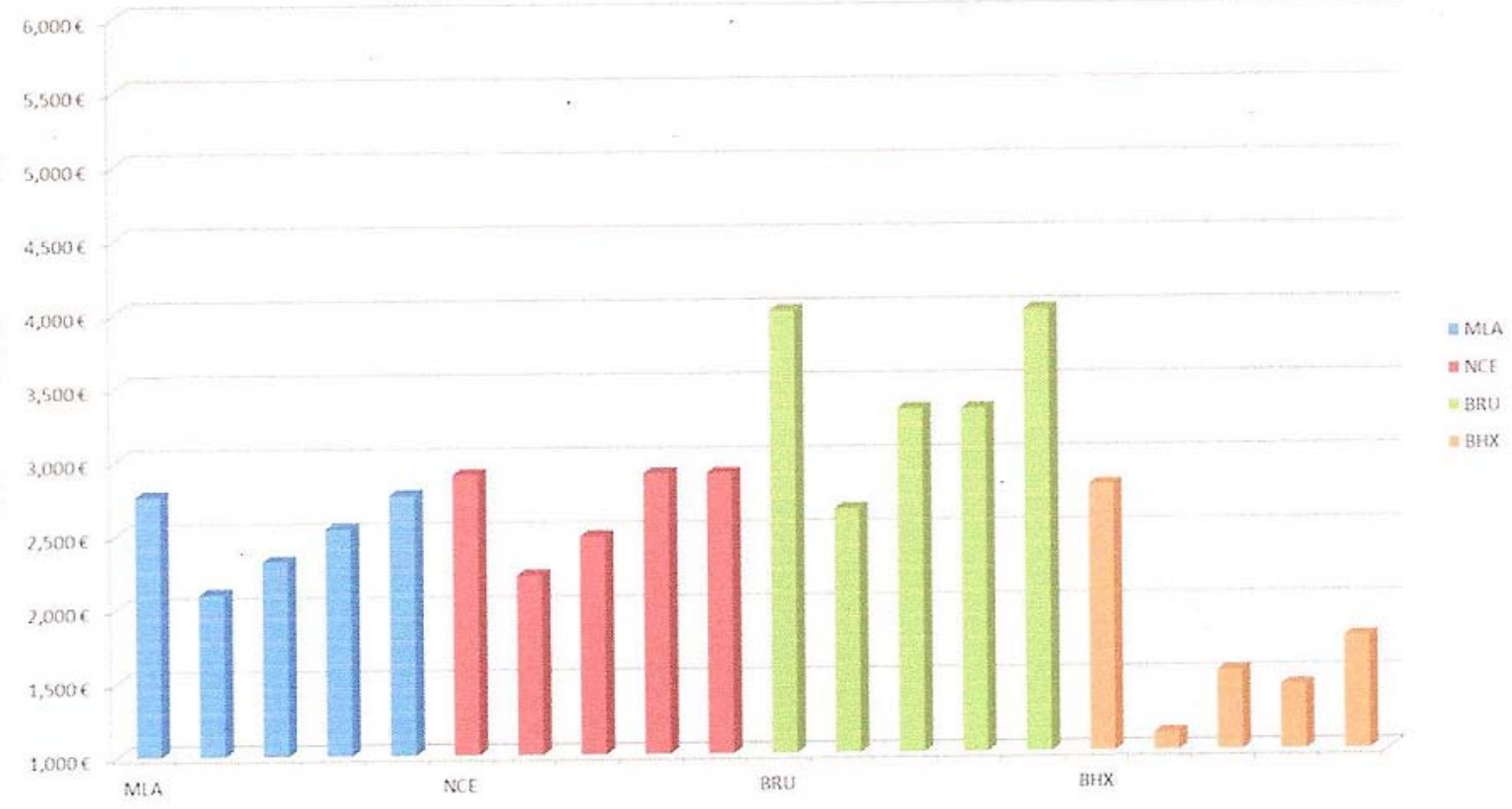

Figure 7: Total Charges for a FR B737-800 operating with a "strategic" incentive scheme, over a 4 year period, including a standard benchmark 